\title{
SUPPRESSION OF OSCILLATIONS IN GODUNOV'S METHOD FOR A RESONANT NON-STRICTLY HYPERBOLIC SYSTEM*
}

\author{
LIN LONGWEI ${ }^{\dagger}$, BLAKE TEMPLE $^{\ddagger}$, AND WANG JINGHUA $§$
}

\begin{abstract}
We prove the stability of the $2 \times 2$ Godunov numerical method in a resonant nonlinear system of conservation laws. The system we study provides one of the simplest settings in which wave speeds can coincide in a nonlinear problem, namely, an inhomogeneous equation in which the inhomogeneity is treated as an unknown variable: $u_{t}+f(a, u)_{x}=0, a_{t}=0$. This is a model for resonance in more complicated systems, such as transonic flow in a variable area duct, and certain resonant problems in multiphase flow and elasticity. We show that the total variation of the conserved quantities can grow at most linearly in weak solutions generated by the $2 \times 2$ Godunov method, under the assumption that $a(x)$ satisfies the "threshold" smoothness condition that the total variation of $a^{\prime}(x)$ is finite. We show by counterexample that the condition is sharp in the sense that there is no bound on the growth rate based on the $C^{1}$ norm of this variable. This is the most complicated setting in which the stability of the $2 \times 2$ Godunov method has been demonstrated, and our results provide the first such result for a numerical method that is (essentially) based on the solution of the Riemann problem for a resonant non-strictly hyperbolic system.

The solution of the Riemann problem is more interesting when wave speeds coincide because the coordinate system of Riemann invariants is singular, and there exists a multiplicity of possible time asymptotic wave patterns for solutions. As a consequence, numerical methods that are based on the solution of the Riemann problem can introduce spurious oscillations in the approximate solutions. Indeed, counterexamples show that the total variation in $u$ of the waves in the Riemann problem step of the $2 \times 2$ Godunov method can tend to infinity as $\Delta x \rightarrow 0$, even when the initial total variation in $u$ is zero. Thus our results verify that the averaging step in the Godunov method "wipes out" the numerical oscillations that can occur in the Riemann problem solution step of the method. We interpret this as demonstrating that numerical methods based on the Riemann problem are viable in this non-strictly hyperbolic setting.
\end{abstract}

Key words. oscillations, Godunov method, resonance

AMS subject classifications. 65M10, 76N99, 35L65, 35L67

1. Introduction. We discuss the issue of "blowup" (we use the term loosely) in the total variation norm for solutions of the $2 \times 2$ resonant nonlinear system of conservation laws

$$
a_{t}=0
$$

$$
u_{t}+f(a, u)_{x}=0
$$

* Received by the editors December 3, 1992; accepted for publication (in revised form) December $1,1993$.

$\dagger$ Department of Mathematics, Zhongshan University, Peoples Republic of China. This research was supported in part by a National Science Foundation US-China Cooperative Research Grant INT-8911134, and by the Institute of Theoretical Dynamics, University of California-Davis, Spring 1990.

$\ddagger$ Department of Mathematics, University of California, Davis, Davis California 95616 (jbtemple@ucdavis.edu). This research was supported in part by National Science Foundation Grant INT-8911134 and DMS-8613450 (Principle Investigator), and by the Institute of Theoretical Dynamics, University of California-Davis.

$\S$ Institute of Systems Science, Academia Sinica, Beijing, Peoples Republic of China. This research was supported in part by a National Science Foundation US-China Cooperative Research Grant number INT-8911134, DMS-8657319, and by the Institute of Theoretical Dynamics, University of California-Davis, Winter 1990. 
(2)

$$
a(x, 0)=a_{0}(x) \equiv a(x)
$$

$$
u(x, 0)=u_{0}(x),
$$

where $u \in R, a \in R$; and we let $U=(a, u), F(U)=(0, f(U))$. System (1) is a system with the two wave speeds $\lambda_{0}(U)=0$ and $\lambda_{1}(U)=\partial f / \partial u$ and is resonant at a state where the two wave speeds coincide, which makes (1) a nonstrictly hyperbolic system. This is the special case of an $n \times n$ resonant nonlinear system as introduced in [10], [11]. (Motivations for this point of view can be found in the work of Marchesin and Paes-Leme; see for example [18].) Note that for fixed $a$, system (1) is equivalent to the inhomogeneous scalar conservation law $u_{t}+f(a(x), u)_{x}=0$. Examples of resonant systems of this general form have been used to model problems in multiphase flow and are related to problems involving transonic flow in a variable area duct [10], [11]. In this context, the canonical type of behavior occurs in a neighborhood of a state $U_{*}=\left(a_{*}, u_{*}\right)$ where the nonlinear wave family is genuinely nonlinear, the nonlinear wave speed is zero at $U_{*}$, and the flux function $f$ is monotone in $a$ at $u_{*}$ [10], [11]. Thus we assume

$$
\begin{gathered}
\lambda_{1}\left(U_{*}\right)=0, \\
\frac{\partial}{\partial u} \lambda_{1}\left(U_{*}\right) \neq 0, \\
\frac{\partial}{\partial a} f\left(U_{*}\right) \neq 0 .
\end{gathered}
$$

These are generic conditions that generalize to the case when $u$ is a vector as well, and they imply that solutions of the linearized equations blow up as $t$ tends to infinity (cf. [10], [11]). Here we prove the stability of the $2 \times 2$ Godunov numerical method by demonstrating that the total variation in $u$ of the approximate solutions generated by this method can grow at only a linear rate under the condition that the function $a(x)$ satisfies the threshold smoothness condition $\operatorname{Var}\left\{a^{\prime}(\cdot)\right\}<\infty$. (We let $\operatorname{Var}\{f(\cdot)\} \equiv$ $\int\left|f^{\prime}(x)\right| d x$ denote the total variation of the function $f$, a measure of the size of the derivative of $f$.) This condition was first identified by Tveito and Winther in [23]. As far as we know, the linear growth rate of $\operatorname{Var}\{u(\cdot, t)\}$ is a new result for the solutions of system (1) as well. ${ }^{1}$

Our analysis relies on the previous work of the authors [15] where we showed that for the nonlinear problem, $\operatorname{Var}\{z(\cdot, t)\}$ remains bounded for all time in solutions generated by the $2 \times 2$ Godunov method, where $z=z(a, u)$ is the variable that defines the singular transformation first introduced by Temple in [22]. Since $z$ is related to $u$ by a singular transformation, bounds on $\operatorname{Var}\{z(\cdot, t)\}$ do not imply bounds on the total variation of the conserved quantity $u$. Examples show that in the nonlinear problem, $\operatorname{Var}\{u(\cdot, t)\}$ can initially grow at an arbitrary rate when $a$ is taken to be of bounded variation [22]. Our result is that when $\operatorname{Var}\left\{a^{\prime}(\cdot)\right\}<\infty$, the quantity $\operatorname{Var}\{u(\cdot, t)\}$

\footnotetext{
${ }^{1}$ In the strictly hyperbolic regime, $\operatorname{Var}\{u(\cdot, t)\}$ can be bounded by a constant times $\operatorname{Var}\{U(\cdot, 0)\}$; but since this fails in the resonant regime (cf. [22]), it is natural to look for a bound on the growth rate for $\operatorname{Var}\{u(\cdot, t)\}$ in the resonant regime.
} 
can grow at only a linear rate, the rate depending only on $\operatorname{Var}\left\{a^{\prime}(\cdot)\right\}, \operatorname{Var}\{a(\cdot)\}$, and $\operatorname{Var}\left\{z_{0}(\cdot)\right\}$. The above example shows that there does not exist a growth rate for $\operatorname{Var}\{u(\cdot, t)\}$ that depends only on $\operatorname{Var}\{a\}$ even when $u_{0}(x) \equiv 0$ [22]. In the next section we also show by counterexample that there does not exist a rate depending only on the $\operatorname{Var}\{a(\cdot)\}$ and the $C^{1}$-norm of $a(x)$. We understand this as follows: the $2 \times 2$ Glimm and Godunov methods are based on approximating solutions locally by time asymptotic states, and the total variation of $u$ in the time asymptotic states is not bounded by the total variation of the initial data. Thus, the total variation in $u$ can initially "blow up" in the total variation norm due to the possible formation of oscillations. Such oscillations can appear after finite time due to the formation of intermediate wave patterns that will later interact and decay, and correspondingly, they can also appear as numerical oscillations in any numerical method based on time asymptotic wave patterns (for example, the Glimm and Godunov methods). Therefore, in terms of the numerical method, our results verify that the averaging step in the Godunov method wipes out the numerical oscillations that can occur in the Riemann problem solution step of the method. We interpret this as demonstrating that numerical methods based on the Riemann problem are viable in this non-strictly hyperbolic setting. Moreover, this is the most complicated setting in which the stability of the $2 \times 2$ Godunov method has been demonstrated, and our results provide the first such stability result for a numerical method based on the solution of the Riemann problem for a resonant non-strictly hyperbolic system.

In terms of the nonlinear problem itself, we interpret our results (as well as the earlier result in [23]) as showing that when $a(\cdot)$ is sufficiently smooth, i.e., $\operatorname{Var}\left\{a^{\prime}(\cdot)\right\}<$ $\infty$, the rate at which the time asymptotic states are taken on in a solution is controlled. This effect is quantified by the statement that $\operatorname{Var}\{u(\cdot, t)\}$ grows at no more than a linear rate when $\operatorname{Var}\left\{a^{\prime}(\cdot)\right\}<\infty$.

Note that the system obtained by linearizing the flux function $F(U)=(0, f(a, u))$ about a state $U_{*}$, where $U_{*}=\left(a_{*}, u_{*}\right)$ satisfies (3)-(5). In this case, letting $U=U_{*}+\bar{U}$, we write $F(U)=F\left(U_{*}\right)+d F\left(U_{*}\right) \bar{U}+$ h.o.t., where h.o.t. are higher order in $|\bar{U}|$, and by $(3)-(5)$,

$$
d F=\left[\begin{array}{ll}
0 & 0 \\
\beta & 0
\end{array}\right],
$$

where $\beta=(\partial f / \partial a)\left(U_{*}\right) \neq 0$. Thus the linearized equations for $\bar{U}$ are

$$
\bar{U}_{t}+\left[\begin{array}{ll}
0 & 0 \\
\beta & 0
\end{array}\right] \bar{U}_{x}=0
$$

which have the solution $\bar{u}=\bar{u}_{0}(x)-\beta \bar{a}^{\prime}(x) t$. Thus in the linearized system, $\bar{u}$ and all $x$-derivatives of $\bar{u}$ also blow up at a linear rate as $t$ tends to infinity.

In $\S 1$, we state our main result on the stability of the Godunov method, we establish notation and review the results in [15], and we show by counterexample that for solutions of $(1), \operatorname{Var}\{u(\cdot, t)\}$ does not grow at a rate depending only on the $\operatorname{Var}\{a\}$ and the $C^{1}$-norm of $a(x)$. The proof of Theorem 2.1 is given in $\S 3$.

2. Preliminaries. In this section we state Theorem 2.1 , our main result on linear growth for $(1),(2)$, and then we establish notation and review the results in [15] that are required for the subsequent proof, which is developed in the next section. At the end of the section we show by counterexample that for solutions of $(1), \operatorname{Var}\{u(\cdot, t)\}$ does not grow at a rate depending only on the $\operatorname{Var}\{a\}$ and the $C^{1}$-norm of $a(x)$; in 
particular, the counterexample indicates that condition $\operatorname{Var}\left\{a^{\prime}(\cdot)\right\}$ is sharp for linear growth. The proof of Theorem 2.1 is postponed until $\S 3$. We first state the main theorem of this paper.

Let $U(x, t)$ be a solution of (1), (2) generated from Godunov's method (to be discussed below) for arbitrary initial data $U_{0}(x) \subset \mathbf{B}$ of compact support, where $\mathbf{B}$ is a neighborhood of $U_{*}$ to be determined below. Assume that $U(x, t)$ is generated from initial data satisfying

$$
\begin{aligned}
& \operatorname{Var}\left\{a^{\prime}\right\} \equiv V_{a}^{\prime}<\infty, \\
& \operatorname{Var}\{a\} \equiv V_{a}<\infty,
\end{aligned}
$$

and

$$
\operatorname{Var}\left\{u_{0}(\cdot)\right\} \equiv V_{u}<\infty
$$

In particular, this implies that (see [22])

$$
\operatorname{Var}_{z}\left\{U_{0}(\cdot)\right\} \equiv V_{z}<\infty \text {. }
$$

Let $C_{f}$ denote the constant

$$
C_{f} \equiv \sup _{\mathbf{B}}\left\{\left|f_{a}\right|,\left|f_{u}\right|,\left|f_{a a}\right|,\left|f_{a u}\right|,\left|f_{u u}\right|\right\}
$$

Our purpose is to prove the following theorem:

THEOREM 2.1. Let $U(x, t)$ be a solution of (1), (2) generated by the $2 \times 2$ Godunov method for arbitrary initial data $U_{0}(x)$, satisfying (8), (9), and (10). Then there exists a constant $C$ depending only on $V_{a}^{\prime}, V_{a}, V_{z}$, and $C_{f}$ such that

$$
\operatorname{Var}\{u(\cdot, t)\} \leq \operatorname{Var}\left\{u_{0}(\cdot)\right\}+C t .
$$

The proof of Theorem 2.1 follows from an analysis of the $2 \times 2$ Godunov numerical method as applied to (1), which we rewrite as the $2 \times 2$ nonstrictly hyperbolic system

$$
U_{t}+F(U)_{x}=0
$$

where $U=(a, u)$ and $F(U)=(0, f(u))$. The Godunov scheme is based on the construction of the Riemann problem for (14). We briefly describe the solution of the Riemann problem as outlined in [15]. The Riemann problem, denoted $\left[U_{L}, U_{R}\right]$, is the initial value problem for initial data given by a jump discontinuity

$$
U_{0}(x)= \begin{cases}U_{L} & \text { if } x<0 \\ U_{R} & \text { if } x>0\end{cases}
$$

Let $\lambda_{i}$ and $R_{i}$ denote the eigenvalues and corresponding unit right eigenvectors of the $2 \times 2$ matrix $d F, i=0,1$. The eigenvalues are given by $\lambda_{0}(U) \equiv 0$ and $\lambda_{1}(U)=\partial f / \partial u$. In [11] it is shown that the assumptions (3)-(5) imply that $\lambda_{1}(U)=0$ defines a smooth curve $\Gamma$ (named the transition curve) in $U$-space passing through the state $U=U_{*}$ in a direction transverse to the $u$-axis, and thus $\Gamma$ is described by a smooth function $u=T(a)$ in a neighborhood of $U_{*}$. The curve $\Gamma$ is the set of states where system (14) 
is non-strictly hyperbolic in a neighborhood of $U_{*}$. By substituting $u-T(a)$ for $u$ in system (14), we obtain an equivalent system in which $\Gamma$ is given by $u=0$; so without loss of generality we assume that in a neighborhood of $U_{*}, \lambda_{1}(U)=0$ if and only if $u=0$. The assumptions (3)-(5) imply that the matrix $d F$ has the Jordan normal form

$$
d F=\left[\begin{array}{ll}
0 & 0 \\
1 & 0
\end{array}\right]
$$

at each state $U \in \Gamma$.

Because (14) is nonstrictly hyperbolic at $U=U_{*}$, there are in general three waves that solve the Riemann problem [15]. The wave curves for (14) are the integral curves of the eigenvector fields $R_{0}$ and $R_{1}$ associated with $\lambda_{0}$ and $\lambda_{1}$. The 1-wave curves are given by $a=\bar{a}, \bar{a}$ constant, and 1-waves are determined by solutions of the scalar conservation law $u_{t}+f(\bar{a}, u)_{x}=0$. The 0 -wave curves are given by $f=$ const. Because of (3)-(5), in a neighborhood of $U_{*}, f=$ const defines a smooth curve of nonzero curvature that is tangent to the curves $a=$ const only at the states $U \in \Gamma$ in the $a u$-plane; and the transition curve $\Gamma$ intersects the 0 -wave and 1 -wave curves transversally at $U_{*}$. To be consistent with [22], [11], [15], we assume without loss of generality that $f_{u u}\left(U_{*}\right)<0$ and $f_{a}\left(U_{*}\right)<0$, so that the curves $f=$ const are convex down in a neighborhood of $U_{*}$ (see Lemma 3.1 in [11] and Figure 1 of [15]). We restrict attention to solutions of (14) that take values in a neighborhood $\mathbf{B}$ of $U_{*}$. Thus let $\mathbf{B}$ denote a neighborhood of $U_{*}$ bounded above by an integral curve of $R_{0}$ and below by an integral curve of $R_{1}$, such that the integral curves of $R_{0}$ are convex down in $\mathbf{B}$, and such that each integral curve intersects the transition curve $\Gamma$ transversally at a unique point in $\mathbf{B}$. Assume further that $\partial f / \partial a \neq 0$ in $\mathbf{B}$. Our assumptions (3)-(5) imply that such an open set $\mathbf{B}$ exists in a neighborhood of $U_{*}$. Under these assumptions, the set $\mathbf{B}$ is an invariant region for Riemann problems for system (1) (cf. [11], [22], [15]):

Proposition 2.2. Let $\mathbf{B}$ denote a neighborhood of $U_{*}$ bounded above by an integral curve of $R_{0}$ and below by an integral curve of $R_{1}$, such that the integral curves of $R_{0}$ are convex down in $\mathbf{B}$, and such that each integral curve cuts the transition curve $\Gamma$ transversally in $\mathbf{B}$. Then $\mathbf{B}$ is an invariant region for Riemann problems in the sense that if $U_{L}, U_{R} \in \mathbf{B}$, then all intermediate states in the solution of the Riemann problem $\left[U_{L}, U_{R}\right]$ are also in $\mathbf{B}$.

We recall also the definition of the singular variable $z$ defined in [22]: for our purpose it suffices to define $z$ by

$$
z(a, u)= \begin{cases}+|f(a, u)-f(a, 0)| & \text { if } U \text { lies to the right of } \Gamma \\ -|f(a, u)-f(a, 0)| & \text { if } U \text { lies to the left of } \Gamma .\end{cases}
$$

(This is slightly different than the definition given in [22], [15], but since $f$ is constant along integral curves of $R_{0}$ and $\Gamma$ is given by $u=0$, the two definitions are essentially equivalent.) Since the curves given by $f=$ const are convex down in a neighborhood of $U_{*}$, we conclude that the mapping

$$
u \rightarrow z
$$

is 1-1 and onto in a neighborhood of $U_{*}$ and is regular except at $\Gamma$ where the Jacobian vanishes. We let $\operatorname{Var}_{z} U_{0}$ denote the total variation of $U_{0}$ in the variable $z$, 
etc. Although for convenience we restrict attention to solutions that take values in a neighborhood $\mathbf{B}$ of $U_{*}$, the results extend globally under straightforward assumptions on $f$.

Now we indicate by counterexample that for solutions of $(1), \operatorname{Var}\{u(\cdot, t)\}$ does not grow at a rate depending only on the $\operatorname{Var}\{a\}$ and the $C^{1}$-norm of $a(x)$ even when $u_{0}(x) \equiv 0$; the counterexample indicates that condition $\operatorname{Var}\left\{a^{\prime}(\cdot)\right\}$ is sharp for linear growth. The counterexample given in [22] shows that for solutions of (14), $\operatorname{Var}\{u(\cdot, t)\}$ does not grow at a rate depending only on $a$ through the $\operatorname{Var}\{a\}$. For this counterexample it suffices to take $a(x)=a_{j}$ for $x_{j} \leq x<x_{j+1}, u_{0}(x) \equiv 0$, where

$$
a_{j}= \begin{cases}a_{*} & \text { if } j \leq 0, \\ a_{*}+\epsilon j / N & \text { if } 0<j \leq N, \\ a_{*}+\epsilon & \text { if } j \geq N,\end{cases}
$$

for some $\epsilon>0$ and $N \in Z$. In this case, $\operatorname{Var}\{u(\cdot, 0+)\}=O(\sqrt{\epsilon N})$ in the exact solution because the wave curves $a=$ const and $f=$ const have a quadratic tangency at $u=0$ (cf. [22]), and thus the growth rate for $\operatorname{Var}\{u(\cdot, 0+)\}$ is not bounded as $N \rightarrow \infty$.

Now consider the initial data $U_{0}^{N}=\left(a_{N}(x), u_{0}^{N}(x)\right)$ where $u_{0}^{N}(x) \equiv 0$ and $a(\cdot) \in$ $C^{1}$ is defined by

$$
a_{N}(x)=a_{*}+\sigma(x) \frac{1}{N} \sin (N x),
$$

where $0 \leq \sigma(x) \leq 1$ is smooth and satisfies $\sigma(x) \equiv 1$ for $0 \leq x \leq 1, \sigma(x) \equiv 0$ for $|x| \geq 2$, and $\left|\sigma^{\prime}(x)\right| \leq 1$. It is easy to verify that

$$
\left|a_{N}^{\prime}(\cdot)\right| \leq 2
$$

and

$$
\operatorname{Var}\left\{a_{N}^{\prime}(x)\right\} \leq 7 .
$$

But when $u=0, f_{u}(a, 0)=0$; so at $t=0,(1)$ reads

$$
u_{t}+f(a, u)_{x}=u_{t}+f_{a} a_{N}^{\prime}
$$

so

$$
u_{t}(x, 0) \approx a_{N}^{\prime}(x)
$$

(where we assume without loss of generality that $f_{a}\left(a_{*}, 0\right)=-1$ ). Thus for $0 \leq x \leq 1$,

$$
u_{t}^{N}(x, 0) \approx \begin{cases}+1 & \text { if } x=\frac{(2 n+1) \pi}{N} \\ -1 & \text { if } x=\frac{2 n \pi}{N}\end{cases}
$$

so on the interval $x \in[0,1]$, the solution $u(x, t)$ satisfies

$$
\operatorname{Var}\{u(\cdot, d t)\} \approx(N / 2 \pi) d t
$$

We conclude that

$$
\frac{d}{d t} \operatorname{Var}\left\{u_{N}(\cdot, 0)\right\} \rightarrow \infty
$$

as $N \rightarrow \infty$, thus verifying that the rate of growth of $\operatorname{Var}\{u(\cdot, t)\}$ is not bounded by a constant depending on $a(x)$ through $\operatorname{Var}\{a(\cdot)\}$ and the $C^{1}$-norm of $a(\cdot)$. 
3. Godunov scheme. In this section we give the proof of Theorem 2.1. We state seven technical lemmas that are required for the proof. The proofs of the first six lemmas are given here, and then we give the proof of Theorem 2.1 as a consequence of the seven lemmas. The proof of Lemma 3.11 is technical and is postponed until after the proof of Theorem 2.1. Now we state and prove the first six lemmas that will be used in the subsequent analysis. To start, let $U_{1}$ and $U_{2}$ be arbitrary states in $\mathbf{B}$, and define the following second-order difference:

$$
\Delta^{2} f\left(U_{2}, U_{1}\right) \equiv \Delta^{2} f_{1,2}=f\left(a_{2}, u_{2}\right)-f\left(a_{2}, u_{1}\right)-f\left(a_{1}, u_{2}\right)+f\left(a_{1}, u_{1}\right) .
$$

LEMmA 3.1. The following estimate holds:

$$
\left|\Delta^{2} f\left(U_{2}, U_{1}\right)\right| \leq O(1)\left\{(\Delta a)^{2}+|\Delta a \Delta z|\right\},
$$

where $\Delta a=a_{2}-a_{1}, \Delta z=\left|z\left(a_{2}, u_{2}\right)-z\left(a_{1}, u_{1}\right)\right|$ and $O(1)$ denotes a generic constant that depends only on $f$ and $\mathbf{B}$.

Proof. By (17),

$$
\Delta^{2} f_{1,2}=-\operatorname{sign}\left\{u_{2}\right\}\left(z\left(a_{2}, u_{2}\right)-z\left(a_{1}, u_{2}\right)\right)+\operatorname{sign}\left\{u_{1}\right\}\left(z\left(a_{2}, u_{1}\right)-z\left(a_{1}, u_{1}\right)\right) \text {. }
$$

Now it suffices to verify (22) in the case that both $u_{1}, u_{2} \leq 0$ or both $u_{1}, u_{2} \geq 0$. To see this, note that

$$
\begin{aligned}
& \Delta^{2} f_{1,2}= f\left(a_{2}, u_{2}\right)-f\left(a_{2}, 0\right)-f\left(a_{1}, u_{2}\right)+f\left(a_{1}, 0\right) \\
&+f\left(a_{2}, 0\right)-f\left(a_{2}, u_{1}\right)-f\left(a_{1}, 0\right)+f\left(a_{1}, u_{1}\right) \\
& \equiv \Delta^{2} f\left(U_{2}, U_{0}^{2}\right)+\Delta^{2} f\left(U_{0}^{1}, U_{1}\right)
\end{aligned}
$$

where $U_{0}^{2} \equiv\left(a_{1}, 0\right)$ and $U_{0}^{1} \equiv\left(a_{2}, 0\right)$. Thus, assuming that we have verified (22) in the above two cases, if $\operatorname{sign}\left\{u_{2}\right\}=-\operatorname{sign}\left\{u_{1}\right\}$, then

$$
\begin{aligned}
\left|\Delta^{2} f_{1,2}\right| & \leq\left|\Delta^{2} f\left(U_{2}, U_{0}^{2}\right)\right|+\left|\Delta^{2} f\left(U_{0}^{1}, U_{1}\right)\right| \\
& \leq O(1)\left\{(\Delta a)^{2}+\left|z_{2} \Delta a\right|\right\}+\left\{(\Delta a)^{2}+\left|z_{1} \Delta a\right|\right\} \\
& \leq O(1)\left\{(\Delta a)^{2}+|\Delta a \Delta z|\right\},
\end{aligned}
$$

where we use the fact that $|\Delta z|=\left|z_{1}\right|+\left|z_{2}\right|$ in this case. Thus we verify (22) in the case that $\operatorname{sign}\left\{u_{1}\right\}=-\operatorname{sign}\left\{u_{2}\right\}$. We do the case $u_{1}, u_{2} \leq 0$. To this end, let $g\left(a, u_{1}, u_{2}\right)$ be the function defined for $u_{1}, u_{2} \leq 0$ by the formula

$$
z\left(a, u_{2}\right)-z\left(a, u_{1}\right)=g\left(a, u_{1}, u_{2}\right)\left\{u_{2}^{2}-u_{1}^{2}\right\} .
$$

We claim that in some neighborhood of $\left(a_{*}, u_{*}, u_{*}\right)$ (we assume this neighborhood consists of the set of all $\left(a, u_{1}, u_{2}\right)$ such that $U, U_{1}, U_{2}$ all lie in $\left.\mathbf{B}\right), g$ is smooth and nonzero and $g^{-1} \equiv \frac{1}{g}$ exists and is smooth in this neighborhood, which intersect the set $u_{1}, u_{2} \leq 0$. First we show that there exists an $\epsilon$ such that $\left|g\left(a, u_{1}, u_{2}\right)\right|>\epsilon$ in this set. To see this, note that since $z\left(a, u_{2}\right)-z\left(a, u_{1}\right)=f\left(a, u_{2}\right)-f\left(a, u_{1}\right)$, we know that if $u_{1} \neq u_{2}$, then $f\left(a, u_{1}\right) \neq f\left(a, u_{2}\right)$ if we choose $\mathbf{B}$ small enough so that $\frac{\partial f}{\partial u} \neq 0$ when $u \neq 0$. Thus when $u_{1} \neq u_{2}$,

$$
g\left(a, u_{1}, u_{2}\right)=\frac{f\left(a, u_{2}\right)-f\left(a, u_{1}\right)}{u_{2}^{2}-u_{1}^{2}} \neq 0 .
$$


Moreover, fixing $u_{1} \neq 0$, we obtain

$$
\frac{\partial f}{\partial u_{2}}=g\left(a, u_{1}, u_{2}\right) 2 u_{2}+g_{u_{2}}\left(u_{2}^{2}-u_{1}^{2}\right)
$$

which implies that when $u_{2}=u_{1} \neq 0$, we have

$$
g\left(a, u_{1}, u_{2}\right)=\frac{1}{2 u_{2}} \frac{\partial f}{\partial u_{1}}\left(a, u_{1}\right) \neq 0
$$

where we assume $\mathbf{B}$ is small enough so that $\partial^{2} f / \partial u^{2} \neq 0$ in $\mathbf{B}$. Finally, for $u_{1}=0$, differentiate $(25)$ to obtain

$$
\frac{\partial^{2} f}{\partial u^{2}}(a, u)=2 g(a, 0, u)+O(u)
$$

which implies that

$$
g(a, 0,0)=\frac{1}{2} \frac{\partial^{2} f}{\partial u^{2}}(a, 0) \neq 0 .
$$

Thus we have that $\left|g\left(a, u_{1}, u_{2}\right)\right|>\epsilon>0$ on compact subsets of $\mathbf{B}$, and hence choosing $\mathbf{B}$ small enough, inside $\mathbf{B}$ itself. The smoothness of $g$ and $g^{-1}$ follow at once from the assumed smoothness of $f$, thus establishing the claim.

To complete the proof of Lemma 3.1, note that

$$
z\left(a_{2}, u_{2}\right)-z\left(a_{2}, u_{1}\right)=g\left(a_{2}, u_{1}, u_{2}\right)\left\{u_{2}^{2}-u_{1}^{2}\right\}
$$

and

$$
z\left(a_{1}, u_{2}\right)-z\left(a_{1}, u_{1}\right)=g\left(a_{1}, u_{1}, u_{2}\right)\left\{u_{2}^{2}-u_{1}^{2}\right\}
$$

thus

(26) $\left|\Delta^{2} f_{1,2}\right|=\left|\left\{g\left(a_{2}, u_{1}, u_{2}\right)-g\left(a_{1}, u_{1}, u_{2}\right)\right\}\left\{u_{2}^{2}-u_{1}^{2}\right\}\right|=O(1)|\Delta a|\left|\left\{u_{2}^{2}-u_{1}^{2}\right\}\right|$.

Moreover, by (24),

$$
\begin{aligned}
u_{2}^{2}-u_{1}^{2}= & g^{-1}\left(a_{2}, u_{1}, u_{2}\right)\left\{z\left(a_{2}, u_{2}\right)-z\left(a_{2}, u_{1}\right)\right\} \\
= & g^{-1}\left(a_{2}, u_{1}, u_{2}\right)\left\{z\left(a_{2}, u_{2}\right)-z\left(a_{1}, u_{1}\right)\right\} \\
& \quad+g^{-1}\left(a_{2}, u_{1}, u_{2}\right)\left\{z\left(a_{1}, u_{1}\right)-z\left(a_{2}, u_{1}\right)\right\} \\
= & O(1) \Delta z+O(1) \Delta a,
\end{aligned}
$$

where we use the fact that $z$ is smooth. Substituting this last line into (26) gives

$$
\left|\Delta^{2} f_{1,2}\right|=O(1)|\Delta a|\{|\Delta a|+|\Delta z|\}=O(1)\left\{\Delta a^{2}+|\Delta a \Delta z|\right\}
$$

which establishes Lemma 3.1.

Let $U=(a, u) \in \mathbf{B}$, and let $a_{1}$ and $a_{2}$ correspond to entries of states in $\mathbf{B}$. Define the following second-order difference:

$$
\Delta^{2} f\left(a_{1}, a_{2}, U\right)=f\left(a_{2}, u\right)-2 f(a, u)+f\left(a_{1}, u\right) .
$$

Lemma 3.2. The following estimate holds:

$$
\left|\Delta^{2} f\left(a_{1}, a_{2}, U\right)\right| \leq O(1)\left\{\left|\Delta a_{1}-\Delta a_{2}\right|+\left|\Delta a_{1}\right|\left|a_{1}-a_{2}\right|\right\},
$$


where $\Delta a_{1}=a-a_{1}$ and $\Delta a_{2}=a_{2}-a$.

Proof. By Taylor's theorem,

$$
\begin{aligned}
\left|\Delta^{2} f\left(a_{1}, a_{2}, U\right)\right|= & \mid\left(a_{2}-a\right) \int_{0}^{1} f_{a}\left(a_{2} \xi+a(1-\xi), u\right) d \xi \\
& +\left(a_{1}-a\right) \int_{0}^{1} f_{a}\left(a_{1} \xi+a(1-\xi), u\right) d \xi \mid \\
= & \mid\left\{\left(a_{2}-a_{1}\right)+\left(a_{1}-a\right)\right\} \int_{0}^{1} f_{a}\left(a_{2} \xi+a(1-\xi), u\right) d \xi \\
& +\left(a_{1}-a\right) \int_{0}^{1}\left\{f_{a}\left(a_{1} \xi+a(1-\xi), u\right) d \xi-f_{a}\left(a_{2} \xi+a(1-\xi), u\right)\right\} d \xi \mid \\
\leq & O(1)\left|\Delta a_{2}-\Delta a_{1}\right|+\sup _{\mathbf{B}} f_{a a}\left|\left(a_{1}-a\right)\left(a_{1}-a_{2}\right)\right|,
\end{aligned}
$$

and the proof is complete.

Our analysis is based on solutions of (14) constructed by the Godunov scheme following the work in [15]. Thus let $U_{\Delta x}(x, t)$ denote an approximate solution of the Cauchy problem (1), (2) generated by the Godunov scheme, for initial data $U_{0}(x)$ taking values in the neighborhood $\mathbf{B}$ of $U_{*}$. Specifically, we discretize $R \times[0, \infty)$ by spatial mesh length $\Delta x$ and time mesh length $\Delta t$ such that

$$
\frac{\Delta t}{\Delta x}=\delta
$$

where $\delta=\operatorname{const}(\lambda)^{-1}$, and

$$
\lambda \equiv \sup _{(a, u) \in \mathbf{B}}\left\{\left|\lambda_{0}\right|,\left|\lambda_{1}\right|\right\}
$$

(It suffices to take $\delta<1 /(2 \lambda)$, but for convenience in the proof of Lemma 3.11, we take $\delta=1 /(4 \lambda)$.) We let $x_{n}=n \Delta x, t_{j}=j \Delta t$ so that $\left(x_{n}, t_{j}\right)$ denote the mesh points of the approximate solution. Define

$$
S_{i}=\left\{(x, t): t_{i} \leq t<t_{i+1}\right\} .
$$

The approximate solution $U_{\Delta x}$ generated by the Godunov scheme is defined as follows [3], [4]: to initiate the scheme at $n=0$, define

$$
U_{j}^{0} \equiv U_{\Delta x}(x, 0)=\frac{1}{\Delta x} \int_{x_{j}}^{x_{j+1}} U_{0}(x) d x, \quad x_{j}<x<x_{j+1} .
$$

Assuming that $U_{\Delta x}(x, t)$ has been constructed for $(x, t) \in \bigcup_{i=0}^{n-1} S_{i}$, we define $U_{\Delta x}$ in $S_{n}$ as the solution of (1) with the initial values

$$
U_{j}^{n} \equiv U_{\Delta x}\left(x, t_{n}\right)=\frac{1}{\Delta x} \int_{x_{j}}^{x_{j+1}} U_{\Delta x}\left(x, t_{n}-\right) d x, \quad x_{j}<x<x_{j+1} .
$$

In other words, at each time $t_{n}$, a piecewise constant approximation $U_{\Delta x}\left(x, t_{n}\right)$ is obtained by taking the arithmetic averages of $U_{\Delta x}\left(x, t_{n}-\right)$ at each interval of the mesh so that the solution in $S_{n}$ can be constructed by solving the Riemann problems $\left[U_{j-1}^{n}, U_{j}^{n}\right]$ posed at each point of discontinuity $\left(x_{j}, t_{n}\right), j \in Z$. The CourantFreidrichs-Levy (CFL) restriction (29) ensures that the Riemann problem solutions in 
each $S_{n}$ do not interact before time $t_{n+1}[22],[15]$. Our results rely on the following theorem, which was proved in [15]:

TheOREM 3.3. Assume that the initial data $U_{0}(x) \in \mathbf{B}$ satisfies the condition $\operatorname{Var}_{z}\left\{U_{0}(\cdot)\right\}=V_{z}<\infty$ and $\operatorname{Var}\{a(\cdot)\}=V_{a}<\infty$. Then $U_{\Delta x}(x, t) \in \mathbf{B}$ for all $x, t \geq 0$, $\operatorname{Var}_{z}\left\{U_{\Delta x}(\cdot, t)\right\}<4 V_{z}$, and a subsequence of $\left\{U_{\Delta x}(\cdot, t)\right\}$ converges boundedly, almost everywhere, to a weak solution of (1), (2) as $\Delta x$ tends to zero.

We use the notation $U_{j-}^{n} \equiv U_{\Delta x}\left(x_{j}+, t_{n}+\right)$ and $U_{j+}^{n}=U_{\Delta x}\left(x_{j+1}-, t_{n}+\right)$, and we let $f_{j}^{n}=f\left(U_{\Delta x}\left(x_{j}, t_{n}+\right)\right), f_{j-}^{n}=f\left(U_{\Delta x}\left(x_{j}+, t_{n}+\right)\right)$, and $f_{j+}^{n}=f\left(U_{\Delta x}\left(x_{j+1}-, t_{n}+\right)\right)$. Here, the symbol + refers to the right side and - the left side of the mesh rectangle $R_{j}^{n}$, which we define by $R_{j}^{n} \equiv\left\{(x, t): x_{j} \leq x<x_{j+1}, t_{n} \leq t<t_{n+1}\right\}$. Using integration by parts it is not difficult to verify that $U_{\Delta x}$ satisfies the difference equation

$$
u_{j}^{n+1}=u_{j}^{n}-\delta\left[f_{j+}^{n}-f_{j-}^{n}\right] .
$$

Let $\Delta u_{j}^{n}=u_{j}^{n}-u_{j-1}^{n}$, so that by (33) we can write

$$
\Delta u_{j}^{n+1}=\Delta u_{j}^{n}-\delta\left[f_{j+}^{n}-f_{j-}^{n}-f_{j-1+}^{n}+f_{j-1-}^{n}\right] .
$$

Our procedure is to estimate the right-hand side of (34), the idea being to add and subtract terms in such a way so as to construct second-order differences of the form (21) and (27), together with a remainder term that forms a collapsing sum in the estimate for $\operatorname{Var}_{u}\left\{U_{\Delta x}\right\}$ given by $\sum_{j=-\infty}^{\infty}\left|\Delta u_{j}^{n+1}\right|$. Since we will be estimating terms at a fixed time level $t_{n}$, we will suppress the index $n$ whenever states are assumed to lie at level $t_{n}$ in an approximate solution $U_{\Delta x}$. Thus we use the notation $f_{i, j}^{-}=$ $f\left(a_{i}, u_{j-}^{n}\right), f_{i, j}^{+}=f\left(a_{i}, u_{j+}^{n}\right)$ and define the first-order differences

$$
\Delta f_{j}^{-}=f_{j, j}^{-}-f_{j, j-1}^{-},
$$

together with the following second-order differences (cf. (21) and (27))

$$
\begin{gathered}
\Delta^{2} f_{i}(u)=f\left(a_{i+1}, u\right)-2 f\left(a_{i}, u\right)+f\left(a_{i-1}, u\right), \\
\Delta^{2} f_{i}(u, v)=f\left(a_{i}, u\right)-f\left(a_{i-1}, u\right)-f\left(a_{i}, v\right)+f\left(a_{i-1}, v\right) .
\end{gathered}
$$

For convenience, we use the following notation for special cases of these second-order differences:

$$
\Delta^{2} f_{i}^{-}=\Delta^{2} f_{i}\left(u_{i-}\right)
$$

$$
\Delta^{2} f_{i}^{+}=\Delta^{2} f_{i}\left(u_{i+}\right),
$$

and

$$
\Delta^{2} f_{i, i-1}^{-}=\Delta^{2} f_{i}\left(u_{i-}, u_{i-1-}\right),
$$

$$
\Delta^{2} f_{i, i-1}^{+}=\Delta^{2} f_{i}\left(u_{i+}, u_{i-1+}\right)
$$


Note that the estimates of either Lemma 3.1 or Lemma 3.2 can be applied to all of the second-order differences above. Our proof of Theorem 2.1 on the stability of Godunov's method and the linear growth of solutions for system (1) proceeds as follows.

Definition 3.4. We say that the types of Riemann problems near mesh point $\left(x_{i}, t_{j}\right)$ fall into case one (we write $\left.T_{j}^{n} \in C_{1}\right)$ if

$$
\left|\Delta f_{j}^{-}\right| \leq(2 \delta)^{-1}\left|\Delta u_{j}^{n}\right|
$$

and

$$
\Delta u_{j}^{n} \Delta f_{j}^{-} \leq 0
$$

If either of these fails, then we say $T_{j}^{n} \in \bar{C}_{1}$.

DEFINITION 3.5. We say that the types of Riemann problems near mesh point $\left(x_{i}, t_{j}\right)$ fall into case two (we write $T_{j}^{n} \in C_{2}$ ) if

$$
\left|\Delta f_{j}^{+}\right| \leq(2 \delta)^{-1}\left|\Delta u_{j}^{n}\right|
$$

and

$$
\Delta u_{j}^{n} \Delta f_{j}^{+} \geq 0
$$

If either of these fails, then we say $T_{j}^{n} \in \bar{C}_{2}$.

Note that the type $T_{j}^{n}$ depends on the wave structures in the Riemann problems posed at $\left(x_{j-1}, t_{n}\right)$ and $\left(x_{j+1}, t_{n}\right)$ as well as $\left(x_{j}, t_{n}\right)$. Theorem 2.1 is a consequence of the following lemmas.

Lemma 3.6. If $T_{j}^{n} \in C_{1}$, then the following estimate holds:

$$
\left|\Delta u_{j}^{n+1}\right| \leq\left|\Delta u_{j}\right|-\delta\left|\Delta f_{j}^{-}\right|+\delta\left|\Delta f_{j+1}^{-}\right|+E_{1}(j),
$$

where

$$
E_{1}(j)=\delta\left|\Delta^{2} f_{j}^{-}\right|+\delta\left|\Delta^{2} f_{j, j-1}^{-}\right|
$$

Proof. By adding and subtracting the appropriate terms to (34) and using the fact that $f_{j}^{+}=f_{j+1}^{-}$, we obtain the identity

$$
\begin{aligned}
u_{j}^{n+1}-u_{j-1}^{n+1}= & \left(u_{j}-u_{j-1}\right)-\delta\left(f_{j+1, j+1}^{-}-f_{j+1, j}^{-}\right)+\delta\left(f_{j, j}^{-}-f_{j, j-1}^{-}\right) \\
& -\delta\left(f_{j+1, j}^{-}-f_{j, j}^{-}\right)+\delta\left(f_{j, j-1}^{-}-f_{j-1, j-1}^{-}\right) \\
& +\delta\left(f_{j, j}^{-}-f_{j-1, j}^{-}\right)-\delta\left(f_{j, j}^{-}-f_{j-1, j}^{-}\right),
\end{aligned}
$$

and this is equivalent to the identity

$$
\Delta u_{j}^{n+1}=\Delta u_{j}-\delta \Delta f_{j+1}^{-}+\delta \Delta f_{j}^{-}-\delta \Delta^{2} f_{j}^{-}-\delta \Delta^{2} f_{j, j-1}^{-} .
$$

Statement (43) follows from (45) by using the inequalities $\Delta u_{j}^{n} \Delta f_{j}^{-} \leq 0$ and $\left|\Delta f_{j}^{-}\right| \leq$ $(2 \delta)^{-1}\left|\Delta u_{j}\right|$ of Definition 3.4.

LEMMA 3.7. If $T_{j}^{n} \in C_{2}$, then the following estimate holds:

$$
\left|\Delta u_{j}^{n+1}\right| \leq\left|\Delta u_{j}\right|+\delta\left|\Delta f_{j-1}^{+}\right|-\delta\left|\Delta f_{j}^{+}\right|+E_{2}(j)
$$


where

$$
E_{2}(j)=\delta\left|\Delta^{2} f_{j-1}^{+}\right|+\delta\left|\Delta^{2} f_{j-1, j-2}^{+}\right|
$$

Proof. By adding and subtracting the appropriate terms to (34) and using the fact that $f_{j}^{+}=f_{j+1}^{-}$, we obtain the identity

$$
\begin{aligned}
u_{j}^{n+1}-u_{j-1}^{n+1}= & \left(u_{j}-u_{j-1}\right)-\delta\left(f_{j, j}^{+}-f_{j, j-1}^{+}\right)+\delta\left(f_{j-1, j-1}^{+}-f_{j-1, j-2}^{+}\right) \\
& -\delta\left(f_{j, j-1}^{+}-f_{j-1, j-1}^{+}\right)+\delta\left(f_{j-1, j-2}^{+}-f_{j-2, j-2}^{+}\right) \\
& +\delta\left(f_{j-1, j-1}^{+}-f_{j-2, j-1}^{+}\right)-\delta\left(f_{j-1, j-1}^{+}-f_{j-2, j-1}^{+}\right),
\end{aligned}
$$

which is equivalent to the identity

$$
\Delta u_{j}^{n+1}=\Delta u_{j}-\delta \Delta f_{j}^{+}+\delta \Delta f_{j-1}^{+}-\delta \Delta^{2} f_{j-1}^{+}-\delta \Delta^{2} f_{j-1, j-2}^{+} .
$$

Thus (46) follows from (48) by using the inequalities $\Delta u_{j}^{n} \Delta f_{j}^{+} \geq 0$ and $\left|\Delta f_{j}^{+}\right| \leq$ $(2 \delta)^{-1}\left|\Delta u_{j}\right|$ of Definition 3.5.

LEMmA 3.8. If $T_{j}^{n} \in C_{1} \cap C_{2}$, then the following estimate holds:

$$
\left|\Delta u_{j}^{n+1}\right| \leq\left|\Delta u_{j}\right|-\delta\left|\Delta f_{j}^{-}\right|-\delta\left|\Delta f_{j}^{+}\right|+E_{3}(j),
$$

where

$$
E_{3}(j)=\delta\left|\Delta^{2} f_{j}\left(u_{j-1-}, u_{j-1+}\right)\right|
$$

Proof. Statement (34) implies the identity

$$
\begin{aligned}
u_{j}^{n+1}-u_{j-1}^{n+1}= & \left(u_{j}-u_{j-1}\right)-\delta\left(f_{j, j}^{+}-f_{j, j-1}^{+}\right)+\delta\left(f_{j, j}^{-}-f_{j, j-1}^{-}\right) \\
& +\delta\left(f_{j, j-1}^{-}-f_{j-1, j-1}^{-}\right)-\delta\left(f_{j, j-1}^{+}-f_{j-1, j-1}^{+}\right),
\end{aligned}
$$

which is equivalent to the identity

$$
\Delta u_{j}^{n+1}=\Delta u_{j}-\delta \Delta f_{j}^{+}+\delta \Delta f_{j}^{-}+\delta \Delta^{2} f_{j}\left(u_{j-1-}, u_{j-1+}\right) .
$$

Thus (49) follows from (51) by using the inequalities given in Definitions 3.4 and 3.5 .

Lemma 3.9. For any type $T_{j}^{n}$, the following estimate holds:

$$
\left|\Delta u_{j}^{n+1}\right| \leq\left|\Delta u_{j}\right|+\delta\left|\Delta f_{j-1}^{+}\right|+\delta\left|\Delta f_{j+1}^{-}\right|+E_{4}(j)
$$

where

$$
E_{4}(j)=\delta\left|\Delta^{2} f_{j+1}\left(u_{j-}, u_{j-2+}\right)\right|+\delta\left|\Delta^{2} f_{j}\left(u_{j-2+}\right)\right|+\delta\left|\Delta^{2} f_{j-1}\left(u_{j-2+}\right)\right| .
$$

Proof. Statement (34) implies the identity

$$
\begin{aligned}
u_{j}^{n+1}-u_{j-1}^{n+1}= & \left(u_{j}-u_{j-1}\right)-\delta\left(f_{j+1, j+1}^{-}-f_{j+1, j}^{-}\right)+\delta\left(f_{j-1, j-1}^{+}-f_{j-1, j-2}^{+}\right) \\
& -\delta\left(f_{j+1, j}^{-}-f_{j, j}^{-}\right)+\delta\left(f_{j+1, j-2}^{+}-f_{j, j-2}^{+}\right) \\
& +\delta\left(f_{j-1, j-2}^{+}-f_{j-2, j-2}^{+}\right)-\delta\left(f_{j, j-2}^{+}-f_{j-1, j-2}^{+}\right) \\
& +\delta\left(f_{j, j-2}^{+}-f_{j-1, j-2}^{+}\right)-\delta\left(f_{j+1, j-2}^{+}-f_{j, j-2}^{+}\right),
\end{aligned}
$$


which is equivalent to the identity

$$
\begin{aligned}
\Delta u_{j}^{n+1}= & \Delta u_{j}-\delta \Delta f_{j+1}^{-}+\delta \Delta f_{j-1}^{+}-\delta \Delta^{2} f_{j+1}\left(u_{j-}, u_{j-2+}\right) \\
& -\delta \Delta^{2} f_{j-1}\left(u_{j-2+}\right)-\delta \Delta^{2} f_{j}\left(u_{j-2+}\right) .
\end{aligned}
$$

Thus (52) follows from (54) directly.

COROLlaRY 3.10. The following estimate holds for $l=1, \ldots, 4$ :

$$
\begin{aligned}
E_{l}(j) \leq O(1) & \left\{\left|\Delta a_{j+1}-\Delta a_{j}\right|+\left|\Delta a_{j}-\Delta a_{j-1}\right|\right. \\
& \left.+\left|\Delta a_{j}\right|\left|a_{j+1}-a_{j-1}\right|+\left|\Delta a_{j-1}\right|\left|a_{j}-a_{j-2}\right|\right\} \\
& +O(1)\left\{\left(\left|\Delta a_{j-1}\right|+\left|\Delta a_{j}\right|+\left|\Delta a_{j+1}\right|\right)\left(\operatorname{Var}_{z} U_{j}^{n}+\operatorname{Var}_{z} U_{j-1}^{n}+\operatorname{Var}_{z} U_{j-2}^{n}\right)\right\},
\end{aligned}
$$

where $\operatorname{Var}_{z} U_{j}^{n}$ denotes the total variation in $z$ of the approximate solution $U_{\Delta x}\left(x, t_{j}+\right)$ in the interval $x_{j}-\leq x \leq x_{j+1}+, \Delta a_{j} \equiv a_{j}-a_{j-1}$, and $O(1)$ denotes a generic constant depending only on $f$ and the supnorm bounds on the solution. Note that by Theorem 3.3,

$$
\sum_{k=-\infty}^{+\infty} \operatorname{Var}_{z} U_{k}^{n} \leq 12 V_{z}
$$

Proof. By Lemmas 3.1 and 3.2 of the previous section, it follows that

$$
\left|\Delta^{2} f_{j}(u)\right| \leq O(1)\left\{\left|\Delta a_{j+1}-\Delta a_{j}\right|+\left|\Delta a_{j}\right|\left|a_{j+1}-a_{j-1}\right|\right\}
$$

and

$$
\left|\Delta^{2} f_{j}(u, v)\right| \leq O(1)\left(\left|\Delta a_{j}\right|^{2}+\left|\Delta a_{j}\right|\left|z\left(a_{j}, u\right)-z\left(a_{j-1}, v\right)\right|\right) .
$$

Thus, in particular we have

$$
\left|\Delta^{2} f_{j}^{-}\right| \leq O(1)\left\{\left|\Delta a_{j+1}-\Delta a_{j}\right|+\left|\Delta a_{j}\right|\left|a_{j+1}-a_{j-1}\right|\right\}
$$

and

$$
\left|\Delta^{2} f_{j, j-1}^{-}\right| \leq O(1)\left\{\left|\Delta a_{j}\right|^{2}+\left|\Delta a_{j}\right|\left(\operatorname{Var}_{z} U_{j}^{n}+\operatorname{Var}_{z} U_{j-1}^{n}\right)\right\}
$$

By the same argument, analogous estimates also hold for $\Delta^{2} f_{j, j-1}^{+}, \Delta^{2} f_{j}\left(u_{j-1-}, u_{j-1+}\right)$, and the remaining second-order differences appearing in $E_{1}(j)-E_{4}(j)$. Putting these estimates together and collecting like terms gives (55).

LEMMA 3.11. If $T_{j-1}^{n} \in \bar{C}_{2}$ and $T_{j}^{n} \in \bar{C}_{1}$, then the following estimate holds:

$$
\left|\Delta f_{j-1}^{+}\right|+\left|\Delta f_{j}^{-}\right| \leq E_{5}(j-1)
$$

where

$$
\begin{aligned}
E_{5}(j-1)= & O(1)\left\{\left|\Delta a_{j+1}-\Delta a_{j}\right|+\left|\Delta a_{j}-\Delta a_{j-1}\right|\right. \\
& \left.+\left|\Delta a_{j}\right|\left|a_{j+1}-a_{j-1}\right|+\left|\Delta a_{j-1}\right|\left|a_{j}-a_{j-2}\right|\right\} \\
(62)+ & O(1)\left\{\left(\left|\Delta a_{j-1}\right|+\left|\Delta a_{j}\right|+\left|\Delta a_{j+1}\right|\right)\left(\operatorname{Var}_{z} U_{j}^{n}+\operatorname{Var}_{z} U_{j-1}^{n}+\operatorname{Var}_{z} U_{j-2}^{n}\right)\right\},
\end{aligned}
$$


The proof of Lemma 3.11 requires the analysis of each admissible Riemann problem type $T_{j-1}^{n}, T_{j}^{n}$ when the conditions of Definitions 3.4 and 3.5 simultaneously fail. These conditions depend only on the structure of the waves at the mesh points $\left(x_{i-1}, t_{n}\right)$ and $\left(x_{i}, t_{n}\right)$, and there can be up to three nontrivial waves in the Riemann problems posed at each mesh point. The proof is technical and is postponed until after the proof of Theorem 2.1. As a motivation we comment that one can verify caseby-case that $T_{j-1}^{n} \in \bar{C}_{2}$ and $T_{j}^{n} \in \bar{C}_{1}$ cannot simultaneously hold in any admissible solution of the Riemann problem under the special assumptions that $\Delta a_{j-1}=\Delta a_{j}$ and that the curves $f=$ const are symmetric about the line $u=0$ and invariant under vertical translations in the $a u$-plane. Thus estimate (61) can be viewed as a perturbation of this case.

It is interesting to note that in the proof of Theorem 2.1 to follow, the entropy condition and the choice of admissible solutions of the Riemann problem enter only through estimate (61) together with the results of Theorem 3.3. Thus we can view inequality (61) as giving a nontrivial characterization of the admissibility condition for Riemann problems in this non-strictly hyperbolic setting.

We use (43)--(61) in the proof of the following proposition:

Proposition 3.12. The following estimate holds:

$$
\sum_{k=i}^{j}\left|\Delta u_{k}^{n+1}\right| \leq \sum_{k=i}^{j}\left|\Delta u_{k}^{n}\right|+F_{i}^{1}+F_{j}^{2}+E(k),
$$

where $F_{i}^{1}$ and $F_{j}^{2}$ are defined by

$$
\begin{aligned}
& F_{i}^{1}= \begin{cases}-\delta\left|\Delta f_{i}^{-}\right| & \text {if }(i, n) \in \mathbf{C}_{\mathbf{1}}, \\
+\delta\left|\Delta f_{i-1}^{+}\right| & \text {if }(i, n) \in \overline{\mathbf{C}}_{\mathbf{1}} ;\end{cases} \\
& F_{j}^{2}= \begin{cases}-\delta\left|\Delta f_{j}^{+}\right| & \text {if }(j, n) \in \mathbf{C}_{\mathbf{2}}, \\
+\delta\left|\Delta f_{j+1}^{-}\right| & \text {if }(j, n) \in \overline{\mathbf{C}}_{\mathbf{2}}\end{cases}
\end{aligned}
$$

and $E(k)$, defined as $E(k) \equiv \sum_{l=1}^{5} E_{l}(k)$, satisfies

$$
\begin{aligned}
|E(k)| \leq & O(1)\left\{\left|\Delta a_{j+1}-\Delta a_{j}\right|+\left|\Delta a_{j}-\Delta a_{j-1}\right|\right. \\
& \left.+\left|\Delta a_{j}\right|\left|a_{j+1}-a_{j-1}\right|+\left|\Delta a_{j-1}\right|\left|a_{j}-a_{j-2}\right|\right\} \\
& \left.+O(1)\left(\left|\Delta a_{j-1}\right|+\left|\Delta a_{j}\right|+\left|\Delta a_{j+1}\right|\right)\left(\operatorname{Var}_{z} U_{j}^{n}+\operatorname{Var}_{z} U_{j-1}^{n}+\operatorname{Var}_{z} U_{j-2}^{n}\right)\right\} .
\end{aligned}
$$

Proof. The estimate (66) follows directly from (55) and (62). We prove (63) by induction. To start the induction, note that when $i-j=0,(63)$ reduces to

$$
\left|\Delta u_{i}^{n+1}\right| \leq\left|\Delta u_{i}^{n}\right|+F_{i}^{1}+F_{i}^{2}+\sum_{l=1}^{4} E_{l}(i) .
$$

But estimate (67) follows directly from (43), (46), (49), and (52) (depending on the possible values for $F_{i}^{1}+F_{i}^{2}$ ), and thus (63) holds for $i-j=0$. Now suppose that (63) holds for $i$ and $j$. We show that it holds for $i-1$ and $j$ as well, thus verifying (63) by induction. In fact, by (67),

$$
\left|\Delta u_{i-1}^{n+1}\right| \leq\left|\Delta u_{i-1}^{n}\right|+F_{i-1}^{2}+F_{i-1}^{1}+\sum_{k=1}^{4} E_{k}(i-1) .
$$


Putting this in (63), we obtain

$$
\sum_{k=i-1}^{j}\left|\Delta u_{k}^{n+1}\right| \leq \sum_{k=i-1}^{j}\left|\Delta u_{k}^{n}\right|+F_{i-1}^{1}+F_{j}^{2}+\left(F_{i}^{1}+F_{i-1}^{2}\right)+\sum_{k=i}^{j} E(k)+\sum_{l=1}^{4} E_{l}(i-1) .
$$

Therefore, in order to prove the proposition, it is sufficient to show that

$$
F_{i}^{1}+F_{i-1}^{2} \leq E_{5}(i-1) \text {. }
$$

By definition, $F_{i}^{1}+F_{i-1}^{2}=0$ if either $T_{i} \in \bar{C}_{1}, T_{i-1} \in C_{2}$, or $T_{i} \in C_{1}, T_{i-1} \in \bar{C}_{2}$; and $F_{i}^{1}+F_{i-1}^{2} \leq 0$ if $T_{i} \in C_{1}, T_{i-1} \in C_{2}$. But by (61) of Lemma 3.11, if $T_{i} \in \bar{C}_{1}, T_{i-1} \in \bar{C}_{2}$, then $\left|\Delta f_{i-1}^{+}\right|+\left|\Delta f_{i}^{-}\right| \leq E_{5}(i-1)$. Thus (63) is established.

Proof of Theorem 2.1. Let $U_{\Delta x}$ be an approximate solution generated by the Godunov scheme from compactly supported initial data $U_{0}(\cdot)$ satisfying $(8),(9)$, and (10) and taking values in B. Since $\operatorname{Var}\left\{z_{\Delta x}\right\}(\cdot, t)$ is uniformly bounded, $f\left(u_{\Delta x}\right)(x, t)$ tends to a constant state as $x$ tends to plus or minus infinity; and thus $F_{j}^{1}$ and $F_{j}^{2}$ tend to zero as $|j|$ tends to infinity. Thus taking $i=-\infty$ and $j=+\infty$ in (63) and using (66), we obtain

$$
\begin{aligned}
& \sum_{k=-\infty}^{+\infty}\left|\Delta u_{k}^{n+1}\right| \leq \sum_{k=-\infty}^{+\infty}\left|\Delta u_{k}^{n}\right|+O(1) \sum_{k=-\infty}^{+\infty}\left\{\left|\Delta a_{j+1}-\Delta a_{j}\right|+\left|\Delta a_{j}-\Delta a_{j-1}\right|\right. \\
& \left.+(71) \quad+\left|\Delta a_{j}\right|\left|a_{j+1}-a_{j-1}\right|+\left|\Delta a_{j-1}\right|\left|a_{j}-a_{j-2}\right|\right\} \\
& +O(1) \sum_{k=-\infty}^{+\infty}\left(\left|\Delta a_{j-1}\right|+\left|\Delta a_{j}\right|+\left|\Delta a_{j+1}\right|\right)\left(\operatorname{Var}_{z} U_{j}^{n}+\operatorname{Var}_{z} U_{j-1}^{n}+\operatorname{Var}_{z} U_{j-2}^{n}\right)
\end{aligned}
$$

But

$$
\sum_{k=-\infty}^{+\infty}\left|\Delta a_{k}-\Delta a_{k-1}\right| \leq V_{a}^{\prime} \Delta x
$$

$$
\sum_{k=-\infty}^{+\infty}\left|\Delta a_{k}\right|\left|a_{k+1}-a_{k-1}\right| \leq 2 V_{a}^{\prime} \Delta x \sum_{k=-\infty}^{+\infty}\left|a_{k+1}-a_{k-1}\right| \leq 2 V_{a} V_{a}^{\prime} \Delta x
$$

and

$$
\sum_{k=-\infty}^{+\infty}\left\{\left(\left|\Delta a_{j-1}\right|+\left|\Delta a_{j}\right|+\left|\Delta a_{j+1}\right|\right)\left(\operatorname{Var}_{z} U_{j}^{n}+\operatorname{Var}_{z} U_{j-1}^{n}+\operatorname{Var}_{z} U_{j-2}^{n}\right)\right\} \leq 36 V_{z} V_{a}^{\prime} \Delta x
$$

where we use the inequality

$$
\left|a^{\prime}(x)\right| \leq \operatorname{Var}\left\{a^{\prime}\right\}
$$

which applies because $\operatorname{Var}\{a(\cdot)\}<\infty$ and $\operatorname{Var}\left\{a^{\prime}(\cdot)\right\}<\infty$ imply that $a^{\prime}(x) \rightarrow 0$ as $|x| \rightarrow \infty$. Putting these in (71) and using CFL yields

$$
\sum_{k=-\infty}^{+\infty}\left|\Delta u_{k}^{n+1}\right| \leq \sum_{k=-\infty}^{+\infty}\left|\Delta u_{k}^{n}\right|+C \Delta t
$$


where $C$ depends only on $V_{a}, V_{a}^{\prime}, V_{z}, \delta$, and $C_{f}$. Moreover, (75) applies to any weak solution obtained as a limit of approximate solutions $U_{\Delta x}$, so this completes the proof of Theorem 2.1.

It remains only to give the following proof.

Proof of Lemma 3.11. Lemma 3.11 is proved by a case-by-case study of the possible wave patterns that can occur in any two consecutive Riemann problems $\left[U_{j-2}^{n}, U_{j-1}^{n}\right],\left[U_{j-1}^{n}, U_{j}^{n}\right]$. Each case depends on the number of waves at each mesh point, on whether they are positive or negative speed, and on whether $\Delta a_{j-1}$ and $\Delta a_{j}$ are positive or negative. Recall that there can be at most three waves in each Riemann problem and that these waves alone determine the types $T_{j-1}^{n}$ and $T_{j}^{n}$. Here we will first state and prove a number of estimates (which we reference as Observations) from which estimate (61) can be deduced in each case. We will then verify (61) in three representative cases, the remaining cases being analagous. Since we are at a fixed time level $t_{n}$, we suppress the index $n$, and we let $E_{5}(j-1) \equiv O(1) E_{5}$ where

$$
\begin{aligned}
E_{5}= & \left\{\left|\Delta a_{j+1}-\Delta a_{j}\right|+\left|\Delta a_{j}-\Delta a_{j-1}\right|+\left|\Delta a_{j}\right|\left|a_{j+1}-a_{j-1}\right|+\left|\Delta a_{j-1}\right|\left|a_{j}-a_{j-2}\right|\right\} \\
& +\left\{\left(\left|\Delta a_{j-1}\right|+\left|\Delta a_{j}\right|+\left|\Delta a_{j+1}\right|\right)\left(\operatorname{Var}_{z} U_{j}^{n}+\operatorname{Var}_{z} U_{j-1}^{n}+\operatorname{Var}_{z} U_{j-2}^{n}\right)\right\}
\end{aligned}
$$

and $O(1)$ denotes a generic constant depending only on $f, \delta$, and the supnorm bounds for the solution. For convenience, we allow the constant $O(1)$ to be adjusted upward as we go along.

OBSERVATION 1. If $\left(\Delta f_{j-1}^{+}\right)\left(\Delta f_{j}^{-}\right) \leq 0$, then we have both

$$
\left|\Delta f_{j-1}^{+}\right| \leq O(1) E_{5} \text { and }\left|\Delta f_{j}^{-}\right| \leq O(1) E_{5} .
$$

Proof. If $\left(\Delta f_{j-1}^{+}\right)\left(\Delta f_{j}^{-}\right) \leq 0$, then $\left|\Delta f_{j-1}^{+}\right|+\left|\Delta f_{j}^{-}\right|=\left|\Delta f_{j-1}^{+}-\Delta f_{j}^{-}\right|$and we can write $\Delta f_{j-1}^{+}-\Delta f_{j}^{-}$as a second-order difference as follows:

$$
\begin{aligned}
\Delta f_{j}^{-}-\Delta f_{j-1}^{+}= & \left(f_{j, j}^{-}-f_{j, j-1}^{-}\right)-\left(f_{j-1, j-1}^{+}-f_{j-1, j-2}^{+}\right) \\
= & -\left(f_{j, j-1}^{-}-f_{j-1, j-1}^{-}\right)+\left(f_{j-1, j-2}^{+}-f_{j-2, j-2}^{+}\right) \\
= & \left\{-\left(f_{j, j-1}^{-}-f_{j-1, j-1}^{-}\right)+\left(f_{j-1, j-1}^{-}-f_{j-2, j-1}^{-}\right)\right\} \\
& +\left\{-\left(f_{j-1, j-1}^{-}-f_{j-2, j-1}^{-}\right)+\left(f_{j-1, j-2}^{+}-f_{j-2, j-2}^{+}\right)\right\} \\
= & -\Delta^{2} f_{j-1}\left(U_{j-1-}\right)-\Delta^{2} f_{j-1}\left(U_{j-1-}, U_{j-2+}\right),
\end{aligned}
$$

where we have used the fact that $f_{j, j}^{-}=f_{j-1, j-1}^{+}$. But by Lemma 3.2,

$$
\left|\Delta^{2} f_{j-1}\left(U_{j-1-}\right)\right| \leq O(1) E_{5},
$$

and by Lemma 3.1 ,

$$
\left|\Delta^{2} f_{j-1}\left(U_{j-1-}, U_{j-2+}\right)\right| \leq O(1) E_{5}
$$

so (77) is verified.

As an immediate consequence of (77) we have the following corollary.

Corollary 3.13. If either $\left|\Delta f_{j-1}^{+}\right|$or $\left|\Delta f_{j}^{-}\right|$is bounded by $O(1) E_{5}$, then so is the other one.

The next two observations give cases in which Lemma 3.11 is easily verified. 

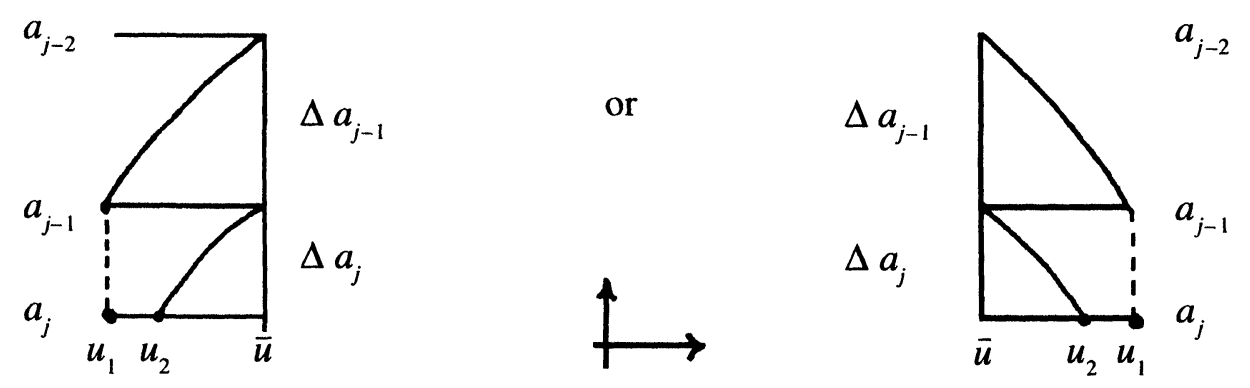

FIG. 1.

OBSERVATION 2. If $\left(\Delta a_{j-1}\right)\left(\Delta a_{j}\right) \leq 0$, then

$$
\left|\Delta a_{j-1}-\Delta a_{j}\right|=\left|\Delta a_{j-1}\right|+\left|\Delta a_{j}\right|=O(1) E_{5} .
$$

Proof. Equation (78) follows directly from the definition of $E_{5}$.

Observation 2 shows that in the case $\left(\Delta a_{j-1}\right)\left(\Delta a_{j}\right) \leq 0$, whenever $\Delta f_{j-1}^{+}$or $\Delta f_{j}^{-}$ are bounded by the first-order differences $\left(\Delta a_{j-1}\right)$ or $\left(\Delta a_{j}\right)$, they are also bounded by the second-order difference $E_{5}$. This makes it easy to verify Lemma 3.11 in the case that the sign of $\Delta a_{j-1}$ is not equal to the sign of $\Delta a_{j}$, so we will omit these cases here.

OBSERVATION 3. If $\left(\Delta u_{j-1}\right)\left(\Delta u_{j}\right) \geq 0$, then when we restrict to the case $\left(\Delta f_{j-1}^{+}\right)$. $\left(\Delta f_{j}^{-}\right) \geq 0$ (the only interesting case by Observation 1$)$, we must have that either

$$
\left(\Delta f_{j-1}^{+}\right)\left(\Delta u_{j-1}\right) \geq 0
$$

or

$$
\left(\Delta f_{j}^{-}\right)\left(\Delta u_{j}\right) \leq 0 .
$$

In this case, to verify Lemma 3.11, it suffices to show that either $\left|\Delta f_{j-1}^{+}\right| \leq$ $(2 \delta)^{-1}\left|\Delta u_{j-1}\right|$ or $\left|\Delta f_{j}^{-}\right| \leq(2 \delta)^{-1}\left|\Delta u_{j}\right|$.

Proof. This follows because the latter estimates verify that either $T_{j-1} \in C^{2}$ or $T_{j} \in C^{1}$ according to Definitions 3.4 and 3.5, either one being contrary to the assumption of Lemma 3.11.

The following observations are more technical.

OBSERVATION 4. Consider any three states $\bar{u}, u_{1}$, and $u_{2}$ in the configuration diagrammed in Fig. 1. Then the following estimate holds:

$$
\begin{array}{r}
\left|f\left(a_{j}, u_{2}\right)-f\left(a_{j}, u_{1}\right)\right| \leq O(1)\left\{\left|\Delta a_{j}-\Delta a_{j-1}\right|+\left|\Delta a_{j}\right|\left|a_{j+1}-a_{j-1}\right|\right. \\
\left.+\left|\Delta a_{j}\right|\left|z\left(a_{j}, \bar{u}\right)-z\left(a_{j-1}, u_{1}\right)\right|\right\} .
\end{array}
$$

Proof. Since $f\left(a_{j}, u_{2}\right)=f\left(a_{j-1}, \bar{u}\right)$ and $f\left(a_{j-1}, u_{1}\right)=f\left(a_{j-2}, \bar{u}\right)$, we can write

$$
\begin{aligned}
f\left(a_{j}, u_{2}\right)-f\left(a_{j}, u_{1}\right)= & f\left(a_{j}, \bar{u}\right)-f\left(a_{j}, u_{1}\right)-f\left(a_{j-1}, \bar{u}\right)+f\left(a_{j-1}, u_{1}\right) \\
& \quad+\left\{-f\left(a_{j}, \bar{u}\right)+2 f\left(a_{j-1}, \bar{u}\right)-f\left(a_{j-2}, \bar{u}\right)\right\} \\
= & -\Delta^{2} f_{j-1}(\bar{u})+\Delta^{2} f_{j}\left(\bar{u}, u_{1}\right) \\
\leq & O(1)\left|\Delta a_{j}-\Delta a_{j-1}\right|+\left|\Delta a_{j}\right|\left|a_{j+1}-a_{j-1}\right| \\
& \quad+O(1)\left|\Delta a_{j}\right|\left|z\left(a_{j-1}, u_{1}\right)-z\left(a_{j}, \bar{u}\right)\right|,
\end{aligned}
$$




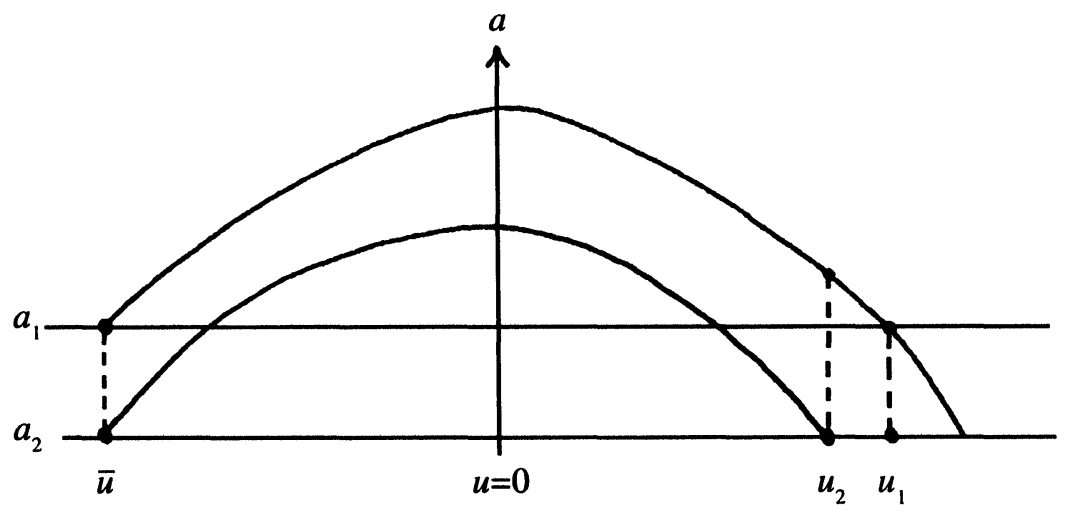

FIG. 2.

where we have applied Lemmas 3.1 and 3.2 in the last line.

OBSERVATION 5. Consider any three states $\bar{u}, u_{1}$, and $u_{2}$ in the configuration diagrammed in Fig. 2, and let $\Delta a \equiv a_{2}-a_{1}$. Then the following estimate holds:

$$
\left|f\left(a_{2}, u_{2}\right)-f\left(a_{2}, u_{1}\right)\right| \leq O(1)\left\{|\Delta a|\left|z\left(a_{2}, \bar{u}\right)\right|\right\} .
$$

Proof. Since we are assuming that the curves $f=$ const are smooth curves that have a quadratic tangency with the curves $a=$ const at each point on the curve $u=0$, for each $a$ and each state $u_{-} \leq 0$ we can define the state

$$
u_{+}=h\left(a, u_{-}\right)
$$

by the condition that $u_{+} \geq 0$ and $f\left(a, u_{-}\right)=f\left(a, u_{+}\right)$. By our assumptions on $f, h$ is smooth and is a 1-1 mapping for each $a$ and

$$
h(a, 0)=0
$$

in our domain. But (81) implies that

$$
\frac{\partial}{\partial a} h(a, 0)=0,
$$

thus we must have

$$
\left|\frac{\partial}{\partial a} h(a, u)\right| \leq O(1)|u| .
$$

Now referring back to Fig. 2, we have $u_{1}=h\left(a_{1}, \bar{u}\right)$ and $u_{2}=h\left(a_{2}, \bar{u}\right)$; thus we can write

$$
\begin{aligned}
f\left(a_{2}, u_{2}\right)-f\left(a_{2}, u_{1}\right) & =f\left(a_{2}, h\left(a_{2}, \bar{u}\right)\right)-f\left(a_{2}, h\left(a_{1}, \bar{u}\right)\right) \\
& =\frac{\partial f}{\partial u}\left(a_{2}, u_{*}\right)\left[h\left(a_{2}, \bar{u}\right)-h\left(a_{1}, \bar{u}\right)\right] \\
& =\frac{\partial f}{\partial u}\left(a_{2}, u_{*}\right) \frac{\partial h}{\partial a}\left(a_{*}, \bar{u}\right) \Delta a .
\end{aligned}
$$




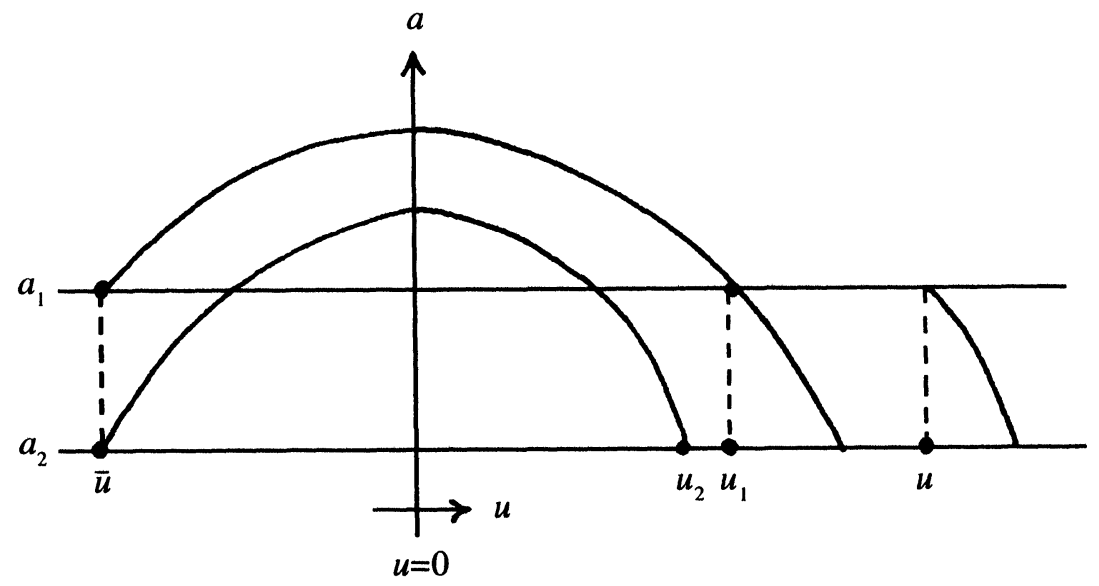

FIG. 3.

But by $(83)$ and the fact that $\frac{\partial f}{\partial u}(a, 0)=0$, we can estimate

$$
\left|\frac{\partial f}{\partial u}\left(a_{2}, u_{*}\right)\right| \leq O(1)|\bar{u}|
$$

and

$$
\frac{\partial h}{\partial a}\left(a_{*}, \bar{u}\right) \leq O(1)|\bar{u}|,
$$

and substituting these into (84), we obtain (80).

The following two corollaries of Observation 5 are useful.

COROLlarY 3.14. Consider any three states $\bar{u}, u_{1}$, and $u_{2}$ in the configuration diagrammed in Fig. 3, and let $u$ be an arbitrary state. Then either

$$
\left|f\left(a_{1}, u\right)-f\left(a_{1}, u_{2}\right)\right| \leq(2 \delta)^{-1}\left|u-u_{1}\right|
$$

or

$$
\left|f\left(a_{1}, u\right)-f\left(a_{1}, u_{2}\right)\right| \leq O(1) E,
$$

where

$$
E=|\Delta a|^{2}+|\Delta a|\left|z\left(a_{1}, u_{1}\right)-z\left(a_{2}, u_{2}\right)\right| .
$$

Proof. In Fig. 3, $u_{1}=h\left(a_{1}, \bar{u}\right)$ and $u_{2}=h\left(a_{2}, \bar{u}\right)$, and we could have $u_{1} \leq u_{2}$ or $u_{2} \leq u_{1}$ (the latter is drawn) and $u$ could be on either side of these. In this case we can estimate

$$
\left|f\left(a_{1}, u\right)-f\left(a_{1}, u_{2}\right)\right| \leq\left|f\left(a_{1}, u\right)-f\left(a_{1}, u_{1}\right)\right|+\left|f\left(a_{1}, u_{1}\right)-f\left(a_{1}, u_{2}\right)\right|,
$$

where

$$
\left|f\left(a_{1}, u\right)-f\left(a_{1}, u_{1}\right)\right| \leq \lambda\left|u-u_{1}\right|
$$

and

$$
\begin{aligned}
\left|f\left(a_{1}, u_{1}\right)-f\left(a_{1}, u_{2}\right)\right| & \leq\left|f\left(a_{2}, u_{1}\right)-f\left(a_{2}, u_{2}\right)\right| \\
& +\left\{\left|f\left(a_{1}, u_{1}\right)-f\left(a_{1}, u_{2}\right)-f\left(a_{2}, u_{1}\right)+f\left(a_{2}, u_{2}\right)\right|\right\} . \\
& \leq O(1) E .
\end{aligned}
$$




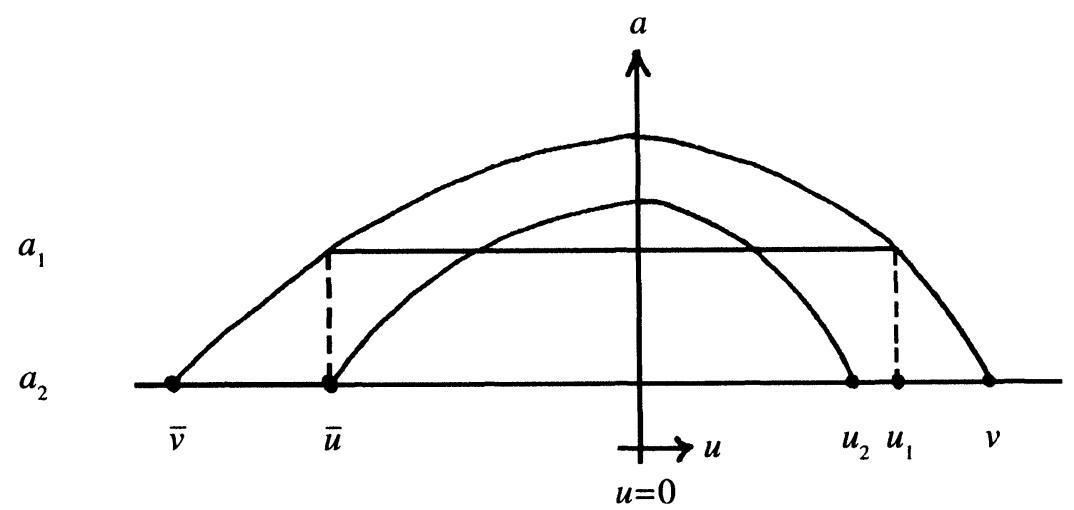

FIG. 4.
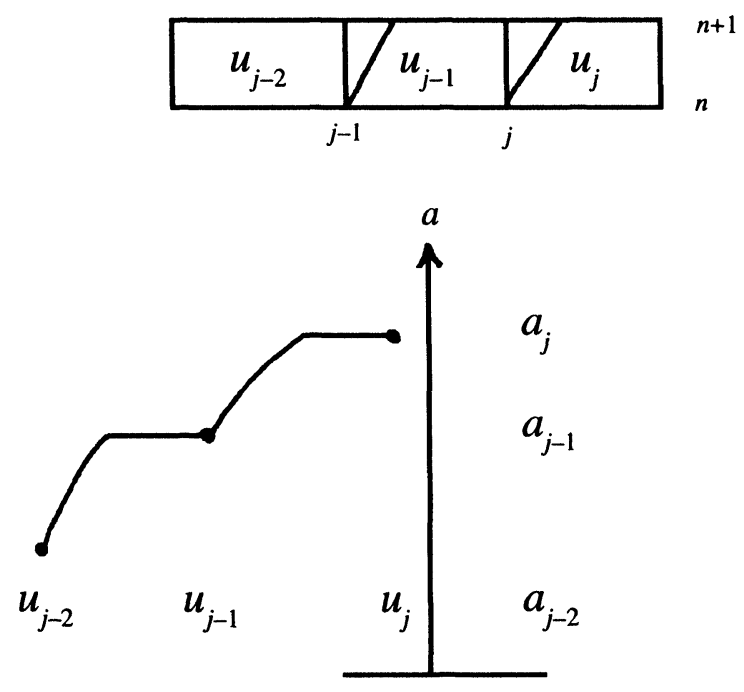

FIG. 5.

In the latter estimate we applied (80) and Lemma 3.1. Thus,

$$
\left|f\left(a_{1}, u\right)-f\left(a_{1}, u_{2}\right)\right| \leq \lambda\left|u-u_{1}\right|+O(1) E .
$$

Therefore, if $\lambda\left|u-u_{1}\right| \geq O(1) E$, then we obtain (85) by taking $\delta=(4 \lambda)^{-1}$, and if $\lambda\left|u-u_{1}\right| \leq O(1) E$, then we obtain (86). This completes the proof of the first corollary. $\quad \square$

Corollary 3.15. Consider any four states $\bar{u}, \bar{v}, u_{1}=h\left(a_{1}, \bar{u}\right)$ and $u_{2}=h\left(a_{2}, \bar{u}\right)$ in the configuration diagrammed in Fig. 4. Then the following estimate holds:

$$
\left|f\left(a_{2}, \bar{u}\right)-f\left(a_{2}, \bar{v}\right)\right|=\left|f\left(a_{2}, u_{1}\right)-f\left(a_{2}, v\right)\right|+O(1)|\Delta a|\left|z\left(a_{2}, \bar{u}\right)\right| .
$$

Proof. By Observation 5 we have that

$$
\left|f\left(a_{2}, u_{2}\right)-f\left(a_{2}, u_{1}\right)\right| \leq O(1)|\Delta a|\left|z\left(a_{2}, \bar{u}\right)\right|,
$$

and since $f\left(a_{2}, \bar{u}\right)-f\left(a_{2}, \bar{v}\right)=f\left(a_{2}, u_{2}\right)-f\left(a_{2}, v\right)$, we can conclude (94) from the triangle inequality. 

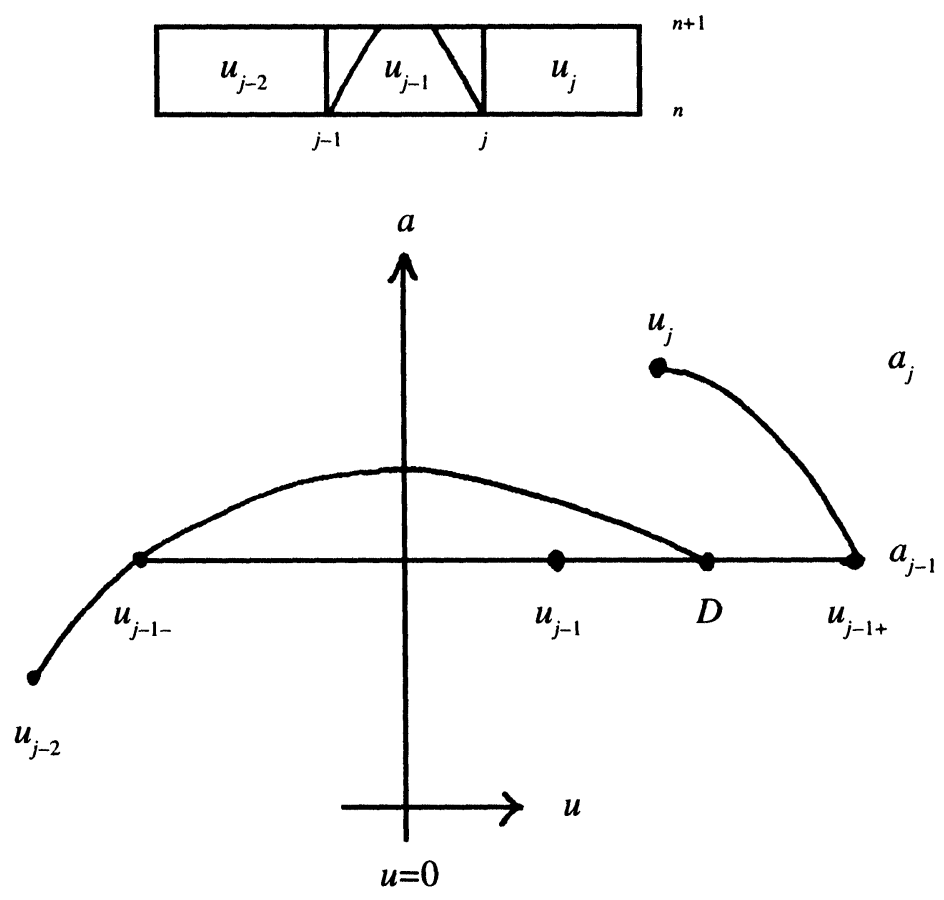

FIG. 6.

Now we verify Lemma 3.11 in three representative cases.

Case (i). We consider the case diagrammed in Fig. 5. In this case there are only positive nonlinear waves at $\left(x_{j-1}, t_{n}\right)$ and $\left(x_{j}, t_{n}\right)$, so $u_{j-1+}=u_{j-1}$ and $u_{j-2+}=u_{j-2}$. Therefore,

$$
\begin{aligned}
\Delta f_{j-1}^{+} & =f\left(a_{j-1}, u_{j-1}\right)-f\left(a_{j-1}, u_{j-2}\right) \\
& =\frac{\partial f}{\partial u}\left(a_{j-1}, u_{*}\right) \Delta u_{j-1} .
\end{aligned}
$$

Thus the inequality

$$
\left|\Delta f_{j-1}^{+}\right| \leq(2 \delta)^{-1}\left|\Delta u_{j-1}^{n}\right|
$$

is evident. In this case it is also straightforward to verify the inequality

$$
\Delta f_{j-1}^{+} \Delta u_{j-1}^{n} \geq 0
$$

so we can conclude that by Definition 3.5, when this wave configuration occurs, $T_{j-1} \in$ $C^{2}$. This contradicts the assumptions of Lemma 3.11, thus verifying Lemma 3.11 in this case. Note that when both of the nonlinear waves have strictly negative speeds, a similar argument shows that $T_{j} \in C_{1}$, again verifying Lemma 3.11.

Case (ii). We consider the case diagrammed in Figs. 6 and 7. In this case there is a nonlinear wave of positive speed at $\left(x_{j-1}, t_{n}\right)$ and a nonlinear wave of negative speed at $\left(x_{j}, t_{n}\right)$, and the waves cross over from the region of positive sound speeds $(u<0)$ to the negative sound speed regime $(u>0)$. We assume here that $\Delta a_{j-1}$ and $\Delta a_{j}$ are both positive. Most of the complications that can occur do occur in this case. 

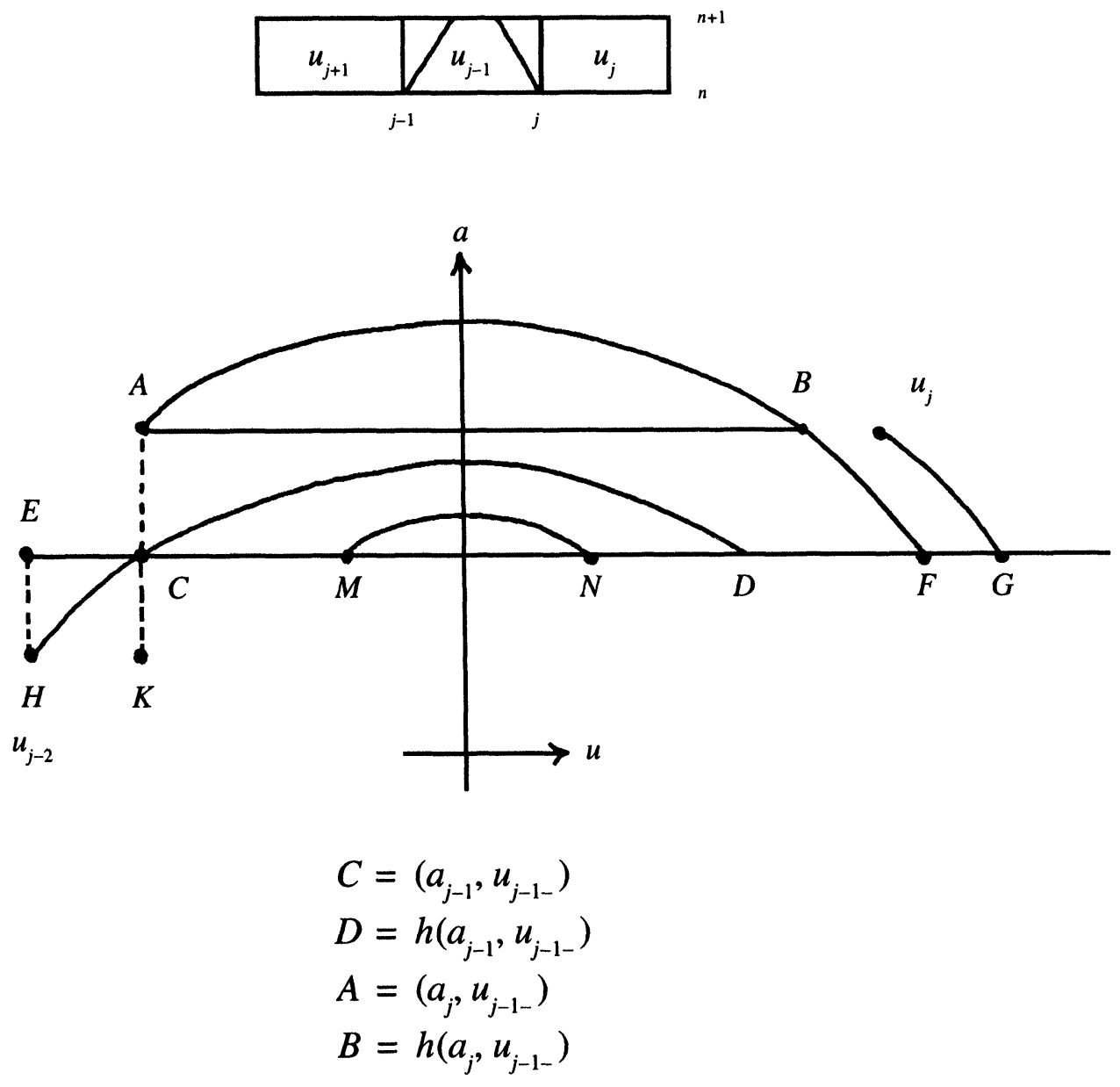

FIG. 7.

(In particular note that in three-wave Riemann problems, the standing wave always ends at $u=0$; this limits the analysis a bit.) Observe first that in order for the left nonlinear wave to be positive, the intermediate state $U_{j-1}=\left(a_{j-1}, u_{j-1}\right)$ must lie to the left of the point labeled D in Figs. 6 and 7. (Recall that the zero speed shocks must jump between equal values of the function $f$.) Here we do the case when $U_{j-1}$ lies between the points labeled $\mathrm{C}$ and $\mathrm{D}$ in Fig. 7, the other case being similar. By Observation 1, it suffices to consider the two cases

$$
\Delta f_{j}^{-} \leq 0 \text { and } \Delta f_{j-1}^{+} \leq 0
$$

or

$$
\Delta f_{j}^{-} \geq 0 \text { and } \Delta f_{j-1}^{+} \geq 0
$$

In case (94), it suffices to verify that either $T_{j} \in C_{1}$ or else both $\left|\Delta f_{j}^{-}\right| \leq O(1) E_{5}$ and $\left|\Delta f_{j-1}^{+}\right| \leq O(1) E_{5}$. But in order for $\Delta f_{j}^{-}=f\left(a_{j}, u_{j}\right)-f(A) \leq 0$, we must have $u_{j}>u(B)$. (See Fig. 7.) In this case, still referring to Fig. 7, it is clear that Corollary 

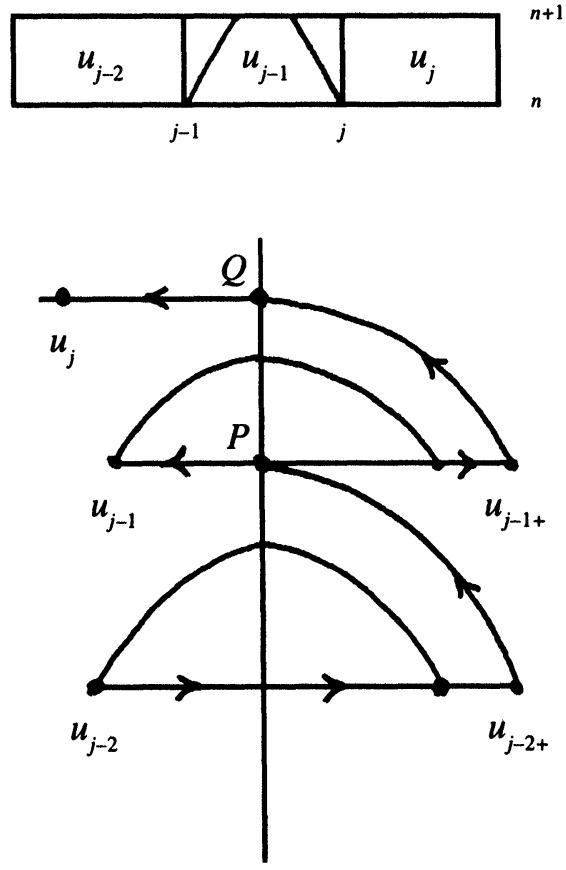

FIG. 8 .

3.14 of Observation 5 applies, and we must have that either $u_{j-1} \leq u_{j}$ and

$$
\left|\Delta f_{j}^{-}\right|=\left|f\left(a_{j}, u_{j}\right)-f(B)\right| \leq(2 \delta)^{-1}\left|u_{j}-u(D)\right| \leq(2 \delta)^{-1}\left|\Delta u_{j}\right|
$$

or else

$$
\left|\Delta f_{j}^{-}\right| \leq O(1) E_{5}
$$

But if $u_{j-1} \leq u_{j}$ and (96) holds, then $T_{j} \in C_{1}$, and we are done; and if (97) holds, then we can apply Corollary 3.13 of Observation 1 to conclude that $\left|\Delta f_{j-1}^{+}\right| \leq O(1) E_{5}$ as well. This verifies Lemma 3.11 under the alternative (94).

Now assume the second alternative, (95), holds. In this case it suffices to show that

$$
\left|\Delta f_{j-1}^{+}\right| \leq(2 \delta)^{-1}\left|\Delta u_{j-1}\right|
$$

since then $T_{j-1} \in C_{2}$. Consider first when $u_{j-1} \leq 0$. (For example, take $\mathrm{M}$ to be $U_{j-1}$ in Fig. 7.) Then, since the nonlinear wave at $x_{j}$ has negative speed, we must also have that $u_{j-1+} \leq u(N)$ in Fig. 7 , so

$$
\begin{aligned}
\left|\Delta f_{j-1}^{+}\right| & \leq|f(E)-f(N)|=|f(E)-f(M)| \\
& \leq(2 \delta)^{-1}\left|\Delta u_{j-1}\right|
\end{aligned}
$$

as required. The inequality (99) is also readily apparent when $u_{j-1} \geq 0$. This completes the proof of Lemma 3.11 in case (ii).

Case (iii). We consider the case diagrammed in Fig. 8. In this case there are two consecutive three-wave Riemann problems at the mesh points $\left(x_{j-1}, t_{n}\right)$ and $\left(x_{j}, t_{n}\right)$. 
We assume again that $\Delta a_{j-1}$ and $\Delta a_{j}$ are both positive. In this case, referring to Fig. 8, we see that both $u_{j-}=0$ and $u_{j-1-}=0$. Thus,

$$
\Delta f_{j}^{-}=f\left(a_{j}, u_{j-}\right)-f\left(a_{j}, u_{j-1-}\right)=0,
$$

and thus $T_{j} \in C_{1}$, contradicting the assumption of Lemma 3.11. Thus Lemma 3.11 holds in this case as well. Note that in this case, $\Delta f_{j-1}^{+}=f\left(a_{j-1}, u_{j-1+}\right)-$ $f\left(a_{j-1}, u_{j-2+}\right)=O(1) E_{5}$ by Corollary 3.13 . This is useful in verifying the cases when only one of the Riemann problem solutions at $x_{j-1}$ and $x_{j}$ have three waves. The remaining cases are omitted.

\section{REFERENCES}

[1] Courant And Freidrichs, Supersonic Flow and Shock Waves, John Wiley \& Sons, New York, 1948.

[2] J. GLimm, Solutions in the large for nonlinear hyperbolic systems of equations, Comm. Pure Appl. Math., 18 (1965), pp. 697-715.

[3] S. K. Godunov, A difference method for numerical calculations of discontinuous solutions of the equations of hydrodynamics, Mat. Sb., 47 (1959), pp. 271-306. (Russian)

[4] - Bounds on the discrepancy of approximate solutions constructed for the equations of gas dynamics, J. Comp. Math. Math. Phys., 1 (1961), pp. 623-637.

[5] E. IsAACSON, Global solution of a Riemann problem for a non-strictly hyperbolic system of conservation laws arising in enhanced oil recovery, Rockefeller University preprint.

[6] E. IsaAcson, D. Marchesin, B. Plohr, And B. Temple, The Riemann problem near a hyperbolic singularity: the classification of solutions of quadratic Riemann problems I, SIAM J. Appl. Math., 48 (1988), pp. 1009-1032.

[7] E. ISAACSON AND B. TEMPLE, The structure of asymptotic states in a singular system of conservation laws, Adv. Appl. Math., 11 (1990), pp. 205-219.

[8] —-, Analysis of a singular system of conservation laws, J. Differential Equations, 65 (1965).

[9] - Examples and classification of non-strictly hyperbolic systems of conservation laws, Abstracts Amer. Math. Soc., January 1985.

[10] - Nonlinear resonance in inhomogeneous systems of conservation laws, Contemp. Math. 108, American Mathematical Society, Providence, RI, 1990.

[11] — Nonlinear resonance in systems of conservation laws, SIAM J. Appl. Anal., submitted.

[12] B. KEYFITZ AND H. KRANZER, A system of non-strictly hyperbolic conservation laws arising in elasticity theory, Arch. Rational Mech. Anal., 72 (1980), pp. 219-241.

[13] P. D. LAX, Hyperbolic systems of conservation laws, II, Comm. Pure Appl. Math., 10 (1957), pp. $537-566$.

[14] P. D. Lax And B. Wendroff, Systems of conservation laws, Comm. Pure Appl. Math., 13 (1960), pp. 217-237.

[15] L. Lin, J. B. TEMPle, AND J. WANG, A comparison of convergence rates for Godunov's method and Glimm's method in resonant nonlinear systems of conservation laws, SIAM J. Numer. Anal., 32 (1995), pp. 838-854.

[16] T. P. LiU, Quasilinear hyperbolic systems, Comm. Math. Phys., 68 (1979), pp. 141-172.

[17] — Resonance for a quasilinear hyperbolic equation, J. Math. Phys., 28 (November 1987).

[18] D. Marchesin And P. J. PAes-Leme, A Riemann problem in gas dynamics with bifurcation, PUC Report MAT 02/84, 1984.

[19] O. A. OleINIK, Discontinuous solutions of non-linear differential equations, Uspekhi Mat. Nauk (N.S.), 12 (1957), pp. 3-73 (Amer. Math. Soc. Transl. Ser. 2, 26, pp. 172-195.)

[20] D. SchaefFer AND M. Shearer, The classification of $2 \times 2$ systems of conservation laws with application to oil recovery, with Appendix by D. Marchesin, P. J. Paes-Leme, D. Schaeffer, and M. Shearer, Comm. Pure Appl. Math., 40 (1987), pp. 141-178.

[21] J. Smoller, Shock Waves and Reaction Diffusion Equations, Springer-Verlag, Berlin and New York, 1980.

[22] B. Temple, Global solution of the Cauchy problem for a class of $2 \times 2$ nonstrictly hyperbolic conservation laws, Adv. in Appl. Math., 3 (1982), pp. 335-375.

[23] A. TVeito AND R. Winther, Existence, uniqueness and continuous dependence for a system of hyperbolic conservation laws modelling polymer flooding, Department of Informatics, University of Oslo, Norway, January, 1990, preprint. 